\title{
1 An inducible AraC that responds to blue light instead of arabinose
}

Edoardo Romano ${ }^{1,2,4,6}$, Armin Baumschlager ${ }^{3,6}$, Emir Bora Akmeriç ${ }^{1,2}$, Navaneethan Palanisamy ${ }^{1,2,4}$, Moustafa Houmani ${ }^{3}$, Gregor Schmidt ${ }^{3}$, Mehmet Ali Öztürk ${ }^{1,2}$, Leonard Ernst ${ }^{5}$, Mustafa Khammash $^{3^{*}}$ and Barbara Di Ventura ${ }^{1,2^{*}}$

${ }^{1}$ Faculty of Biology, Institute of Biology II, University of Freiburg, Freiburg, Germany

${ }^{2}$ Centers for Biological Signalling Studies BIOSS and CIBSS, University of Freiburg, Freiburg, Germany

${ }^{3}$ Department of Biosystems Science and Engineering, ETH Zürich, Basel, Switzerland

${ }^{4}$ Heidelberg Biosciences International Graduate School (HBIGS), University of Heidelberg, Heidelberg, Germany

${ }^{5}$ BioQuant Center for Quantitative Biology, University of Heidelberg, Heidelberg, Germany

${ }^{6}$ These authors contributed equally: E. Romano, A. Baumschlager

* email: mustafa.khammash@bsse.ethz.ch; barbara.diventura@bio.uni-freiburg.de

In Escherichia coli, the operon responsible for the catabolism of L-arabinose is regulated by the dimeric DNA-binding protein AraC. In the absence of Larabinose, AraC binds to the distal $\mathrm{I}_{1}$ and $\mathrm{O}_{2}$ half-sites, leading to repression of the downstream $P_{B A D}$ promoter. In the presence of the sugar, the dimer changes conformation and binds to the adjacent $I_{1}$ and $I_{2}$ half-sites, resulting in the activation of $P_{B A D}$. Here we engineer blue light-inducible AraC dimers in Escherichia coli (BLADE) by swapping the dimerization domain of AraC with blue light-inducible dimerization domains. Using BLADE to overexpress proteins important for cell shape and division site selection, we reversibly control cell morphology with light. We demonstrate the exquisite light responsiveness of BLADE by employing it to create bacteriographs with an unprecedented quality. We then employ it to perform a medium-throughput characterization of $39 \mathrm{E}$. coli genes with poorly defined or completely unknown function. Finally, we expand the 
initial library and create a whole family of BLADE transcription factors (TFs), microbiology.

While the preferred carbon source for E. coli under most conditions is glucose, other sugars, such as lactose or arabinose, also support cell growth, albeit typically at a slower rate $^{1,2}$. Three operons are responsible for the uptake and catabolism of L-arabinose: the $B A D$ operon, encoding three catabolic enzymes that convert L-arabinose to $\mathrm{D}$ xyluluose-5-phosphate; the $F G H$ operon, encoding the transporters that regulate Larabinose uptake when its concentration in the extracellular environment is low, and the araE operon, encoding a low-affinity transporter that acts at high extracellular Larabinose concentrations ${ }^{3,4}$. In the absence of L-arabinose, $\mathrm{P}_{\mathrm{BAD}}$ is repressed by AraC, the regulator of the system, bound to the distal $\mathrm{I}_{1}$ and $\mathrm{O}_{2}$ half-sites, which causes the

43 formation of a DNA loop that sterically blocks the access of the RNA polymerase to 44 the promoter (Fig. 1a). In the presence of $L$-arabinose, transcription from the $\mathrm{P}_{\mathrm{BAD}}, \mathrm{P}_{\mathrm{FGH}}$ and $\mathrm{P}_{\mathrm{E}}$ promoters is activated by $\mathrm{AraC}$, which additionally negatively feeds back on its own promoter $\mathrm{P}_{\mathrm{C}}$, found upstream of, and in reverse orientation to, $\mathrm{P}_{\mathrm{BAD}}{ }^{3,4}$. Activation results from AraC binding to the adjacent $\mathrm{I}_{1}$ and $\mathrm{I}_{2}$ half-sites, which recruits the RNA polymerase (Fig. 1a). AraC is composed of an N-terminal dimerization domain (DD) and a C-terminal DNA binding domain (DBD) connected via a linker (Fig. 1b).

50 Interestingly, AraC is always a homodimer, whether bound to arabinose or not ${ }^{4}$.

51 Binding of arabinose triggers a conformational change in AraC, which results in the 52 two DBDs being oriented in a way that favors their interaction with the $\mathrm{I}_{1}$ and $\mathrm{I}_{2}$ half- 
53 sites rather than the $\mathrm{I}_{1}$ and $\mathrm{O}_{2}$ half-sites (Fig. 1a) $)^{3,4}$. The mechanism explaining this

54 conformational change, which involves ligand-induced regulation of the position of the

$55 \mathrm{~N}$-terminal arm of $\mathrm{AraC}$, has been named the light switch, despite AraC not being a

56 photoreceptor ${ }^{3}$. We reasoned that, if $\mathrm{AraC}$ could be made to respond to light, as

57 previously done for other bacterial and eukaryotic transcriptional regulators ${ }^{5,6}$, it

58 would be possible for microbiologists to reversibly steer, with high spatio-temporal

59 resolution, a great variety of biological processes relying on gene expression. They

60 would simply employ well-known $\mathrm{P}_{\mathrm{BAD}}$-based vectors, such as pBAD33, modified only

61 to express the engineered light-sensitive $\mathrm{AraC}$ in place of the arabinose-sensitive

62 natural one. Importantly, strains previously constructed to control with arabinose a

63 genomic locus, in which the $\mathrm{P}_{\mathrm{BAD}}$ promoter was inserted in place of an endogenous

64 promoter $^{7-9}$, would be fully compatible with this system. Here we show that, by

65 swapping the AraC DD with the blue light-triggered dimerizing protein $\mathrm{VVD}^{10}$, and by

66 selecting the appropriate linker between VVD and the DBD, we are able to render AraC

67 blue light responsive. We characterize this novel AraC, which we name BLADE (for

68 Blue Light-inducible AraC Dimers in E. coli), in terms of kinetics, reversibility, and

69 light dependence. Taking advantage of the ability of BLADE to trigger gene expression

70 only in illuminated cells, we perform bacterial photography and reproduce the Blade

71 Runner movie poster at high resolution using a lawn of bacteria expressing the

72 superfolder green fluorescent protein (sfGFP) under the control of BLADE. We then

73 utilize BLADE to control E. coli cell morphology by overexpressing $\operatorname{MinD}^{\Delta 10}$, MreB

74 and RodZ. Employing a previously constructed E. coli strain where endogenous rodZ

75 is under the control of the $\mathrm{P}_{\mathrm{BAD}}$ promoter $^{7}$, we demonstrate that light, but not arabinose,

76 allows for the reversible switching between round and rod cell morphologies. To

77 showcase the advantage of light as external trigger in medium and high-throughput 

assays, we build a library of 117 constructs to characterize 39 E. coli genes with unknown or poorly defined function in terms of intracellular localization and effect on cell growth and morphology. We investigate the mechanism of BLADE action in vivo, and show that beyond contacting the $\mathrm{I}_{2}$ half-site in the lit state, the dark state involves the formation of aggregates, which likely contribute to the tightness of the system. We engineer an entire family of BLADE TFs creating a much larger library comparing two light-inducible dimerization domains, different linkers and positioning of the components. Interestingly, we find that the order of the light-dependent dimerizing and DBD domains does not need to resemble that of wild type AraC. We show that a synthetic promoter containing two $I_{1}$ half-sites is still light-inducible and leads to higher light/dark fold change in gene expression compared to the wild type $\mathrm{P}_{\mathrm{BAD}}$ promoter based on $\mathrm{I}_{1}-\mathrm{I}_{2}$ in a small range of BLADE concentrations. Finally, we develop a highthroughput characterization approach using a novel 96-well light induction setup, which can be easily built and employed, to find optimal expression levels of the BLADE TFs for best performance. We envision that BLADE will stimulate the incorporation of optogenetic experiments in microbiology due to its compatibility with previously constructed strains and plasmids, its added functionality that cannot be easily achieved with chemical inducers, and its reliable performance.

\section{Results}

\section{Creation of a small library of chimeric VVD-AraC fusion constructs}

Inspired by a previous study in which chimeric AraC constructs have been cloned to probe the role of the $\mathrm{DD}$ and $\mathrm{DBD}^{11}$, we reasoned that, by exchanging the dimerization domain of AraC with a light-inducible dimerization domain (Fig. 1b), we would be able to control with light the switching of this engineered AraC from monomer to dimer 
103 (Fig. 1c). In its monomeric form, the engineered AraC would contact the high-affinity

$104 \mathrm{I}_{1}$ half-site ${ }^{12}$, but not the low-affinity $\mathrm{I}_{2}$ half-site, needed to recruit the RNA polymerase.

105 Its function as a light-inducible TF would depend on finding the appropriate linker 106 supporting the correct orientation of the two DBDs after dimer formation, permissive 107 of $\mathrm{I}_{1}-\mathrm{I}_{2}$ binding (Fig. 1c). As the light-triggered dimerization domain we selected VVD, 108 which has often been successfully employed to control with light the dimerization of 109 proteins of interest ${ }^{5,6,13,14}$. VVD senses blue light via the flavin adenine di-nucleotide 110 (FAD) chromophore ${ }^{10}$. Blue light triggers the formation of a cysteinyl-flavin adduct, 111 which generates a new hydrogen bond network that releases the N-terminus (N112 terminal cap) from the protein core and restructures it creating a new dimerization 113 interface ${ }^{15,16}$. We swapped AraC dimerization domain with $\mathrm{VVD}^{\mathrm{N} 56 \mathrm{~K} / \mathrm{C} 71 \mathrm{~V}}$, a double 114 mutant shown to stabilize the dimer ${ }^{5}$, and cloned seven constructs having different 115 linkers between $\mathrm{AraC}_{\mathrm{DBD}}$ and $\mathrm{VVD}$ (Fig. 1d). We removed the araC gene from 116 pBAD33, and introduced two constitutive promoters of different strength (J23101* and $\left.117 \mathrm{~J} 23101^{* *}\right)$ to drive the expression of the chimeric VVD-AraC $\mathrm{DBD}_{\mathrm{DD}}$ fusion constructs 118 (Supplementary Fig. 1). For a reporter gene, we cloned $m$ Cherry downstream of the

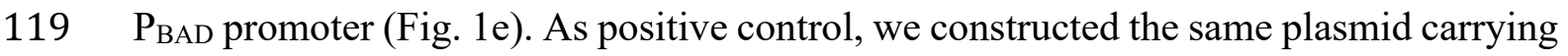
120 full-length AraC in place of the VVD-AraC fusion (Supplementary Fig. 2c), while the 121 plasmid without any TF was constructed to serve as negative control to monitor leaky 122 expression from $\mathrm{P}_{\mathrm{BAD}}$ (Supplementary Fig. 2b). Flow cytometry analysis of $E$. coli 123 MG1655 cells transformed with the small library of VVD-AraC fusions, as well as the 124 negative and the positive controls, kept in the dark or illuminated with $460 \mathrm{~nm}$ light (5 $125 \mathrm{~W} / \mathrm{m}^{2}$ ) for 4 hours showed that all $14 \mathrm{VVD}$-AraC constructs were light-inducible, 126 despite being less optimal than full-length $\mathrm{AraC}$ (Fig. 1f and Supplementary Fig. 3). 127 Different linkers corresponded to different amounts of gene expression. With the 
128

129 its activation was well tolerated by the cells (Supplementary Fig. 4).

\section{Characterization of BLADE}

141 A clear advantage of light as an external trigger is that it can be easily turned off,

142 enabling reversible control of gene expression, without the need of potentially 143 damaging and time-consuming washing steps. We exposed E. coli MG1655 cells 144 transformed with pBLADE-mCherry to alternating 2 hours-cycles of blue light and 145 darkness, for a total of 3 illumination cycles and measured mCherry levels via flow 146 cytometry at the end of every phase. The same expression levels were reached after 147 every illumination phase, and the reporter was repressed to the same extent after every 148 dark phase (Fig. 1g). Next, we measured the kinetics of mCherry expression from 149 pBLADE and found that the half-maximum was reached after about 2.5 hours of 150 induction with light, while the levels plateaued after 5 hours of light induction (Fig.

151 1h). To assess the requirement of BLADE in terms of blue light, we measured mCherry 
152 levels obtained at different light intensities, and found that $1 \mathrm{~W} / \mathrm{m}^{2}$ was sufficient to

153 obtain reporter gene expression levels close to the saturation value (Fig. 1i).

154 Next we sought to demonstrate that BLADE is useful to control the expression of

155 functional E. coli proteins, and not only fluorescent reporters. We thus cloned eyfp-

$156 \min D$ into pBLADE. MinD is a dynamically membrane-bound ATPase, which,

157 together with MinC and MinE, constitutes the Min system, a machinery needed to place

158 the divisome at mid-cell ${ }^{17}$ and to aid chromosome segregation ${ }^{18}$. In order to exert its

159 inhibitory function against FtsZ, the protein that starts divisome assembly, MinC must

160 be recruited to the cytoplasmic membrane by $\operatorname{MinD}^{19,20}$. In E. coli, MinD - and,

161 consequently, MinC - oscillate from pole to pole due to the action of MinE, with a

162 period of 50-60 seconds at room temperature ${ }^{21}$. When averaged over time, MinC/D

163 concentration is highest at the poles and minimal at mid-cell, causing the septum to

164 form at mid-cell. Overexpression of MinD results in filamentation, because endogenous

165 MinE is not sufficient to displace all MinD molecules from the membrane, allowing

166 the MinCD complex to become stably and homogenously membrane-bound, inhibiting

167 FtsZ everywhere ${ }^{17}$. Thus, MinD is a good candidate to check the tightness of the

168 BLADE system. Time-lapse fluorescence microscopy showed that eYFP-MinD

169 oscillations were present only in cells illuminated with blue light and not in those kept

170 in the dark (Supplementary Fig. 5a and Supplementary Video 1). The distribution of

171 the cell length for both non-induced and induced samples was comparable to that of the

172 same strain transformed with the negative control (Supplementary Fig. 5b).

\section{Spatial control of gene expression}

175 One of the benefits of optogenetic induction is the ability to modulate gene expression

176 in a spatially dependent fashion. To showcase how BLADE could be used to control 

expression of a target gene only in selected cells, we cloned sfGFP 22 into pBLADE. $E$. coli MG1655 cells transformed with pBLADE-sfGFP were then applied to an agar pad and subjected to confocal microscopy to expose a limited area $\left(6.4 \mu \mathrm{m}^{2}\right)$ to blue light every 5 minutes. After 3 hours, sfGFP was expressed up to 6.7 -fold more in the illuminated cells compared to the surrounding non-illuminated cells (Supplementary Fig. 6). Another interesting application of light-inducible TFs that relies on the possibility to shine light on a plate in desired patterns, is bacterial photography ${ }^{23}$. To assess the effectiveness of BLADE in this type of application, we covered a lawn of $E$. coli MG1655 cells transformed with pBLADE-sfGFP with a photomask depicting the Blade Runner movie poster (Fig. 2a). We illuminated the plate with blue light overnight and then took several microscopy pictures and stitched them together (Fig. 2b). The sensitive light response of BLADE yielded a good contrast, resulting in a high quality bacteriograph that allowed for the faithful reproduction of the details in the poster, such as facial expressions (Fig. 2c).

\section{Controlling $E$. coli cell morphology with BLADE}

Cell morphology impacts growth and survival in diverse environments ${ }^{24,25}$. Being able to generate desired cell morphologies with light could pave the way to performing experiments to better understand the contribution of cell morphology to bacterial fitness and adaptation to a particular environment. To demonstrate light control of cell morphology, we selected three E. coli proteins to overexpress: $\mathrm{MinD}^{\Delta 10}$, MreB and RodZ. MinD ${ }^{\Delta 10}$ is a truncated form of MinD lacking the last 10 amino acids constituting the membrane targeting sequence (MTS). Without the MTS, MinD ${ }^{\Delta 10}$ cannot associate with the membrane and remains cytoplasmic. It however maintains the ability to homodimerize ${ }^{26}$. We hypothesized that $\operatorname{MinD}^{\Delta 10}$ could heterodimerize with 
endogenous MinD. The heterodimer formed by MinD and $\mathrm{MinD}^{\Delta 10}$ would not be able to stably bind to the membrane, because a monovalent MTS is not sufficient for this ${ }^{27}$.

204 With MinD sequestered into the cytoplasm, endogenous MinC would no longer be 205 recruited to the membrane, and FtsZ should be free to start divisome assembly at the 206 poles, leading to the formation of anucleated mini-cells and variably long cells (Fig. 3a), which would manifest as a multimodal cell length distribution in a population of cells. MreB is the bacterial actin homolog, necessary for the establishment and 209 maintenance of rod shape and cell wall synthesis ${ }^{28-30}$. Its assembly is regulated by 210 RodZ, a transmembrane protein that binds MreB, altering the conformational dynamics

211 and intrinsic curvature of MreB polymers ${ }^{31-33}$. It has been previously established that overexpression of MreB or RodZ leads to cell elongation and thickening ${ }^{30,31,34}$. We

213 cloned the above-mentioned genes into pBLADE and transformed each into MG1655

214 E. coli cells. We then exposed the cells to 4 hours of blue light illumination. Cells kept 215 in the dark served as controls. BLADE-induced $\mathrm{MinD}^{\Delta 10}$ overexpression led to the 216 formation of minicells; cells kept in the dark were indistinguishable from those 217 transformed with an empty pBLADE, which served as negative control (Fig. 3b, c).

218 The phenotype was not caused by the illumination (Fig. $3 \mathrm{c}$ ). In contrast to $\mathrm{MinD}^{\Delta 10}$ 219 overexpression, BLADE-induced $\mathrm{MreB}$ and RodZ overexpression led to cell 220 elongation and thickening, while cells kept in the dark were indistinguishable from the 221 negative control (Fig. 3d-f). We additionally controlled endogenous RodZ with 222 BLADE using a previously constructed strain (KC717), where the endogenous 223 promoter driving rodZ expression has been exchanged with $\mathrm{P}_{\mathrm{BAD}}{ }^{7}$. In the absence of 224 arabinose, the endogenous chromosomal copy of AraC inhibits transcription from $\mathrm{P}_{\mathrm{BAD}}$, 225 thus RodZ is not expressed and cells are spherical ${ }^{7,31,32,35}$. In the presence of arabinose, 226 endogenous AraC initiates transcription from $\mathrm{P}_{\mathrm{BAD}}$ and, consequently, RodZ is 
expressed, leading to the reappearance of rod-shaped cells ${ }^{7}$. We transformed $\mathrm{KC} 717$ cells either with pBLADE-RodZ (population A) or with an empty pBAD33 deprived

229 of $\operatorname{araC}$ and $\mathrm{P}_{\mathrm{BAD}}(\mathrm{pBAD \#}$; population $\mathrm{B}$ ) and kept both populations either uninduced

230 (in the dark for population A, and without arabinose for population B) or induced them

231 for 4 hours (with blue light for population A and with arabinose for population B) (Fig.

$2323 \mathrm{~g})$. At this time point, population A recovered the rod-shape to a greater extent than

233 population B (Fig. 3h). To showcase the power of optogenetics to quickly switch

234 induction off, we subjected the cells to a recovery phase, by putting them into the dark

235 (population A) and washing arabinose off (population B). While it was possible to 236 obtain spherical cells again after 2 hours of dark incubation, the cells that had been 237 induced with arabinose did not recover the initial phenotype and rather became even 238 more rod-shaped (Fig. 3h).

Characterization of $E$. coli genes with unknown function in terms of intracellular

241 localization and effect on growth and morphology

242 The E. coli genome contains 4623 genes, 35\% of which currently lack experimental

243 evidence of function ${ }^{36}$. Since light is particularly well-suited for medium to high-

244 throughput studies due to its low cost, scalability, and effortless application, we used

245 BLADE to characterize some of these genes in terms of their intracellular localization

246 and effect on cell growth and morphology. We randomly selected 34 completely

247 uncharacterized genes and included 5 additional genes, for which some information

248 was available: $y d a T$, which was shown to lead to cell elongation and reduced survival

249 when overexpressed in E. coli ${ }^{37} ; y d i Y$, which was shown to be induced by acid and was 250 predicted to be an outer membrane protein ${ }^{38} ; y c b K$ (renamed MepK), which was shown 251 to be a murein hydrolase involved in cell wall synthesis ${ }^{39} ;$ yehS, whose downregulation 
has been shown to improve the growth of $E$. coli in n-butanol and n-hexane ${ }^{40}$; and $y e b E$,

253 which was shown to be induced by copper in a CpxA/CpxR-dependent manner ${ }^{41}$, and

254 was predicted to be localized to the inner membrane ${ }^{42}$. Importantly, fluorescence

255 microscopy-based localization studies have not been so far carried out for any of these

25639 genes. Since fusion to a fluorescent protein (FP) could alter or impair the function

257 of the gene products, we cloned each of the 39 genes in native form into pBLADE (Fig.

258 4a). However, in order to monitor the localization of the gene products in E. coli cells,

259 we additionally cloned for each gene N- and C-terminal fusions to sfGFP (Fig. 4a). We

260 tested both termini because it is known that the terminus at which the FP is fused often

261 plays a role in determining whether the fusion protein maintains the same localization

262 as the native one ${ }^{43-45}$. In total, we constructed a library of 117 plasmids. Those bearing

263 the native genes were subjected to growth assays and differential interference contrast

264 (DIC) microscopy, while those bearing the fusions to sfGFP were subjected to

265 fluorescence microscopy (Fig. 4a). Before performing experiments, we applied

266 bioinformatics and computational structural biology approaches to predict the function

267 and localization of the 39 selected genes (Fig. 4a). We used three different tools

268 (Argot2.5 $5^{46}$, PANNZER2 ${ }^{47}$, and DeepGoPlus ${ }^{48}$ ) that predict protein function and

269 localization from amino acid sequence information only and one protein 3D modeling

270 tool $\left(\right.$ Phyre $\left.2^{49}\right)$ that uses this information as well as secondary structure prediction to

271 find a template structure that best represents the submitted protein for $3 \mathrm{D}$ modeling

272 (Supplementary Table 1). We generated a consensus table for localization and function

273 taking the predictions shared by at least two out of the four methods (Supplementary

274 Table 2). A consensus was found for 14 out of 39 genes for functional prediction and

275 for 21 out of 39 for localization prediction. We first analyzed the effect of

276 overexpressing the native proteins on bacterial growth. We found six genes whose 
products significantly affected the growth of MG1655 cells: three positively (yahC, $y e b E$ and $y e b Y$ ) and three negatively (yhhM, yjeO and ypaB; Fig. 4b and Supplementary

279 Fig. 7a). Interestingly, yebY is predicted to have transaminase activity (Supplementary 280 Table 2), which could explain why cells overexpressing it grow faster. To assess if any 281 of the 39 genes caused morphological changes, we performed DIC microscopy on MG1655 cells exposed to light for 4 hours. While most genes did not cause morphological alterations, two led to cell elongation ( $y d a T$ and $y d h L)$ and one to cell lysis $(y h c F$; Fig. 4c). Our results thus confirm previous observations on the effect of $285 y d a T$ overexpression on cell morphology ${ }^{37}$, and further indicate that $y d h L$ may be 286 involved in cell division. Since $y d c F$ overexpression caused cell death in this assay, we 287 additionally measured the $\mathrm{OD}_{600}$ of the cell culture after 4 hours of growth in the incubator and found that it was indeed reduced compared to that of the cultures overexpressing $y d a T$ and $y d h L$ as well as compared to cells transformed with empty pBLADE (Supplementary Fig. 7b). Notably, $y h c F$ did not cause growth defects in the 291 assay performed in the 96-well plate. However, it is known that bacteria grow slower in a 96-well plate than in a flask, due to the lower oxygen exchange and shaking.

293 Therefore, it cannot be excluded that the 6 genes found to affect growth in the 96 well294 plate assay form only a partial list, and that other genes among the selected 39 may also 295 affect $E$. coli growth when overexpressed.

296 To study the localization of the uncharacterized genes, we performed fluorescence 297 microscopy. As expected, not all gene products tolerated fusions to either terminus 298 (Supplementary Table 2). For some, fluorescence was barely detectable for one of the 299 two fusions, and for others the localization was not the same for both fusions 300 (Supplementary Fig. 8a, b and Supplementary Table 2). All in all, we found 3 genes 301 whose products co-localized with the nucleoid, 14 that co-localized to the cytoplasmic 
membrane, and 26 that formed foci (Fig. 4d). While the localization alone is not

303 sufficient to reveal the function of the 39 genes, it gives important information and, for

304 some of the genes, suggests a potential mechanism of action. For instance, $y d a T$, which

305 is reported to be a toxin ${ }^{37}$, may exert this function by binding and inhibiting DNA

306 gyrase, since we found it co-localized on the nucleoid. Other toxins that inhibit DNA

gyrase and co-localize to the nucleoid have been described ${ }^{50,51}$.

The mechanism of BLADE-mediated blue light-inducible gene expression involves

311 Wild type AraC and BLADE are substantially different in their mode of action: AraC

312 is always a dimer that, in the absence of arabinose, binds the $\mathrm{I}_{1}$ and $\mathrm{O}_{2}$ half-sites and,

313 in the presence of the sugar, binds the $I_{1}$ and $I_{2}$ half-sites (Fig. 1a). In contrast, BLADE

314 is monomeric in the dark and dimeric under blue light illumination (Fig. 5a). It is hard 315 to predict the 3D structure of the light-induced BLADE dimer. In principle, dimeric

316 BLADE could assume a conformation resembling either that of the AraC dimer free of

317 arabinose, or that of the arabinose-bound dimer, or even a different conformation not

318 found in the natural protein that would nonetheless favor interaction with $\mathrm{I}_{1}$ and $\mathrm{I}_{2}$. All

319 the data we obtained strongly suggest that dimeric BLADE preferentially assumes a 320 conformation that leads to its interaction with the $I_{1}$ and $I_{2}$ half-sites. In particular, the 321 increase in reporter gene expression after illumination can be explained only if BLADE 322 contacts the $\mathrm{I}_{2}$ half-site and recruits the RNA polymerase at the $\mathrm{P}_{\mathrm{BAD}}$ promoter. The $\mathrm{I}_{1}$ 323 half-site is likely contacted also by monomeric BLADE, given that a single DBD of 324 AraC was shown to bind to it in vitro ${ }^{12}$. However, this is not sufficient for recruiting 325 the RNA polymerase to the $\mathrm{P}_{\mathrm{BAD}}$ promoter $^{52}$. To further prove that the $\mathrm{I}_{2}$ half-site is 
contacted in vivo by BLADE in the presence of blue light, we constructed a modified

327 pBLADE plasmid, in which the $\mathrm{I}_{2}$ half-site was cloned in inverse orientation, while

328 keeping the -35 region of the $\mathrm{P}_{\mathrm{BAD}}$ promoter untouched (Supplementary Fig. 9a). In this

329 case, there was no significant difference in mCherry levels between dark and light 330 samples (Supplementary Fig. 9b).

331 In Neurospora crassa, the organism in which it is naturally expressed, VVD is actually

332 degraded in the dark $^{53-55}$. We asked whether VVD may trigger the degradation of

333 BLADE in E. coli cells in the dark, which could add another layer of regulation to the

334 system and contribute to its tightness. To address this question, we fused sfGFP to the

335 C-terminus of BLADE to avoid any interference with dimerization and performed flow

336 cytometry to measure BLADE levels in cells kept in the dark and exposed to blue light

337 for 4 hours. The sfGFP levels were comparable in both conditions (Fig. 5b). However,

338 fluorescence microscopy revealed the presence of bright fluorescent foci in half of the

339 cells kept in the dark (Fig. 5c and Supplementary Fig. 9c), while less than $20 \%$ of the

340 illuminated cells showed foci (Fig. 5d and Supplementary Fig. 9c). To investigate the

341 nature of the foci, we performed fluorescence recovery after photobleaching (FRAP)

342 experiments and found the foci to be static (Supplementary Fig. 9d), suggesting they

343 are aggregates rather than functional liquid droplets. It has been previously shown that

344 VVD transitions between locally unfolded and folded states and that light shifts the

345 half-life of the transition from about 5 minutes to 6 hours $^{56}$. It was suggested that

346 simultaneous unfolding of several structural elements of VVD could lead to

347 aggregation in the dark ${ }^{56}$. The aggregates we observed in E. coli could, therefore, be

348 due to the VVD moiety in BLADE. To prove that the aggregates are related to the light

349 response of VVD, we mutated the adduct-forming cysteine to alanine (VVD ${ }^{\mathrm{C} 108 \mathrm{~A}}$ )

350 within BLADE. We expected the mutant to show aggregates also under blue light 
351 illumination, since $\mathrm{VVD}^{\mathrm{C} 108 \mathrm{~A}}$ is not responsive to light. Indeed $40 \%$ of the cells

352 presented aggregates both in the dark and when illuminated with blue light for 4 hours

353 (Fig. 5e and Supplementary Fig. 9c).

354 Finally, to investigate whether light leads to the dispersion of previously formed foci,

355 we performed time-lapse fluorescence microscopy to follow individual foci over time

356 in illuminated cells. If light actively disperses the aggregates, foci in individual cells

357 should disappear. Alternatively, the aggregates may remain intact under blue light

358 illumination, but form less frequently in newborn cells. We found that the aggregates

359 do not disperse, but are instead asymmetrically segregated during cell division

360 (Supplementary Video 2). Newborn cells contain either no foci or foci much smaller

361 than those found in cells kept in the dark (Supplementary Video 2).

363 Expanding the family of BLADE TFs

364 In principle, BLADE could have been designed using other light-inducible dimerization

365 domains. Moreover, the position of this domain with respect to the DBD of AraC may

366 not need to reflect that found in the wild type protein. To test if other functional

367 combinations with different characteristics could be identified, we generated a much

368 larger set of samples for characterization, with a library size significantly larger than

369 the one described earlier. As a light-inducible dimerization unit, we included not only

370 VVD, but also the Light Oxygen Voltage (LOV) domain of Vaucheria frigida

371 Aureochrome1 (VfAu1) ${ }^{57,58}$, which is naturally found C-terminally to a bZip DBD ${ }^{58}$,

372 and which, like VVD, homodimerizes upon blue light stimulation ${ }^{59,60}$. To assess the

373 functionality of the chimeric transcription factors (cTFs), we used only the $\mathrm{P}_{\mathrm{BAD}}$ 
promoter ( $\mathrm{I}_{1}-\mathrm{I}_{2}$ half-sites) and removed the upstream regulatory elements $\left(\mathrm{O}_{1}\right.$ and $\mathrm{O}_{2}$ half-sites $)^{61}$. Since the results with the initial VVD-AraC fusions showed that the strength of the constitutive promoter driving their expression played an important role

377 in determining the light/dark fold change (Fig. 1f), we systematically explored how the 378 expression levels of the cTF affected mCherry levels in the dark and after blue light 379 illumination. To this aim, we first used an isopropyl- $\beta$-D-thiogalactopyranoside 380 (IPTG)-inducible promoter ${ }^{62}$ to achieve various levels of expression of the cTF, with 381 the goal of finding the most appropriate expression level. This identification of 382 appropriate or 'optimal' cTF expression levels to achieve a certain output (e.g. highest 383 fold change, or certain levels of dark or light-induced expression) is the first step in our 2-step method (Fig. 6a). For this, an IPTG-inducible promoter is used to cover a wide range of cTF concentrations - a step that only requires a single genetic construct. The second step maps the transcriptional strength of the IPTG induction levels to 387 constitutive promoters. This step only needs to be performed once for a given inducible 388 promoter. Using constitutive promoters allows for future uses of the optimized systems 389 and eliminates the need for inducer molecules. We employed the IPTG-inducible 390 promoter as part of single-plasmid systems that can be assembled in one-pot Golden

391 Gate cloning reactions comprised of easily adaptable components. This allows for the 392 characterization of these and other cTFs by enabling the exchange of every functional 393 genetic component (Supplementary Fig. 10). In principle, one may expect that different 394 possible scenarios could arise from the influence of the cTF concentration on the output expression (Supplementary Fig. 11). For example, Scenario 1 represents the case in

396 which the higher the cTF concentration, the higher the output will be, both in the dark 397 and after illumination, maintaining the fold change relatively constant. Scenario 2 398 represents the case in which a concentration threshold exists, after which there is a 
reduction in the light-induced fold change of the output. This effect could be due, for example, to resource limitations in the cells that express the cTF and the output gene. Scenario 3 corresponds to a different effect—one that also implies the existence of optimal intermediate cTF concentrations. Here, high cTF concentrations do not alter the output expression in the light, but they instead cause the dark state to increase, for instance due to the formation of dimers in the dark. We used a wide range of inducer concentrations to capture these potential scenarios, and focused on the output light/dark fold change, an important feature of light-inducible proteins. Depending on the application, other properties such as high output expression or low dark state might be more relevant. To characterize many individual samples, under the same light input conditions, a novel high-throughput light induction device was needed. We therefore developed a light induction device which can be used for standard 96-well microtiter plates in which the light input for every well can be steered individually (Fig. 6b). The setup comprises a custom-made printed circuit board (PCB) with 96 individual light emitting diodes (LEDs) of three different wavelengths (red, green and blue). Each LED can be controlled individually using a microcontroller, enabling the exposure of each well to the same light intensity, a crucial aspect for the characterization of the cTFs. A milled metal plate placed in between the PCB and the LEDs dissipates the heat produced by the light induction device. A 3D-printed microplate adapter on top of the metal plate allows for the precise positioning of the 96-well plate. The high-throughput characterization allowed us to calculate IPTG dose-response curves of the same construct receiving the same light input as well as grown in the dark (Fig. 6a). In addition, it allowed us to test the $\mathrm{N}$ - and $\mathrm{C}$-terminal positioning of the light-inducible dimerization unit, as well as different linkers connecting the two domains, which would not have been feasible without this technical setup (Fig. 6c). 
424 For this characterization we used E. coli strain BW25113 $\triangle a r a C$ from the KEIO

425 collection $^{63}$, and integrated lacYA177C into the attB site for facilitated IPTG

426 diffusion ${ }^{30}$. We first tested the constructs in the library with variable order between

$427 \mathrm{AraC}_{\mathrm{DBD}}$ and the light-inducible dimerization unit, but constant linker. Our 96-well

428 light induction setup allowed us to use a wide range of IPTG concentrations, from no

429 induction to a concentration of 2 mM IPTG. For all constructs, the highest fold change

430 was reached at intermediate mCherry expression levels (indicated with red lines in two

431 examples shown in Fig. 6d). Placing $\mathrm{AraC}_{\mathrm{DBD}}$ at the $\mathrm{C}$-terminus led to higher fold

432 changes for VVD-based constructs, mainly due to lower mCherry expression in the

433 dark (Fig. 6e). For VfAu1, the opposite was true (Fig. 6f). Next, we investigated the

434 effect of linker length on the cTFs. Based on the results obtained with the first library,

435 we placed $\mathrm{AraC}_{\mathrm{DBD}} \mathrm{C}$-terminally for the VVD-based constructs, and N-terminally for

436 those based on VfAu1. We selected a set of linkers from a previous report ${ }^{64}$.

437 Additionally, we cloned a linker-free variant for each of the dimerization domains,

438 where the domains where directly fused with each other. We found that linker lengths

439 from zero to 7 amino acids gave rise to the highest fold change for both photosensors

440 (Fig. 6g, h). All these functional fusions expand the family of BLADE TFs.

441 Since in future biological applications BLADE should be constitutively expressed to

442 dispense of the use of any chemical inducer, as was the case in our initial experiments,

443 we aimed to find constitutive promoters that would give rise to expression levels

444 comparable to those obtained with various IPTG concentrations. We therefore cloned

445 in the same plasmid bearing the IPTG-inducible promoter a library of constitutive

446 promoters ${ }^{65}$, as well as an additional weaker constitutive promoter variant to cover very

447 low expression levels. To minimize the potential influence of individual promoters on

448 mRNA transcription and translation initiation, we used a ribosome binding site (RBS) 
containing an insulating ribozyme (RiboJ) ${ }^{66}$. Plotting the mCherry fluorescence levels

450 obtained with the constitutive promoters and with the IPTG-inducible promoter at

451 different IPTG concentrations in the same plot, it is possible to find the constitutive

452 promoter that best matches the expression from the IPTG-inducible one at the desired

453 IPTG concentration (Fig. 6a). While the characterization was performed in an E. coli

454 strain in which the arabinose operon was deleted, the results do not change if a strain 455 with endogenous $a r a C$ is used (Supplementary Fig. 12).

A synthetic $P_{B A D}$ promoter comprising two $I_{1}$ half-sites is light-inducible and achieves higher light/dark fold changes

459 We performed the same systematic characterization of BLADE family members using 460 a synthetic $\mathrm{P}_{\mathrm{BAD}}$ promoter, where the weak affinity $\mathrm{I}_{2}$ half-site was exchanged with a 461 second copy of the high affinity $I_{1}$ half-site (Supplementary Fig. 13). This synthetic 462 promoter is insensitive to arabinose, as it is constitutively active when used with wild 463 type $\mathrm{AraC}^{67}$. We asked whether it could be made light-inducible instead. Results with 464 this promoter were consistent with those obtained with the synthetic $\mathrm{P}_{\mathrm{BAD}}$ promoter 465 consisting only of the $I_{1}$ and $I_{2}$ half-sites (Fig. $6 \mathrm{j}, \mathrm{k}$ ), confirming our findings regarding 466 the position of the domains and the linkers within the cTFs. Interestingly, the maximal 467 dark/light fold change for the same cTFs was higher compared to that obtained with the $468 \mathrm{I}_{1}-\mathrm{I}_{2}$ synthetic promoter. High IPTG concentrations led to toxic amounts of mCherry 469 expression and were, therefore, indistinguishable for dark and light induction in most 470 cases. Careful adjustment of the cTF concentration is required to achieve the desired 471 light inducibility, which then unlocks an expression system with an even higher 472 expression level and fold change than the one based on the original $\mathrm{I}_{1}$ and $\mathrm{I}_{2}$ half-sites. 


\section{Discussion}

474 AraC is among the best studied bacterial transcriptional regulators, and the $\mathrm{P}_{\mathrm{BAD}}$ 475 promoter is one of the inducible promoters most often employed in microbiology and synthetic biology. We have developed an entire family of AraC-derived TFs, which we

477 call BLADE, that activate transcription from the $\mathrm{P}_{\mathrm{BAD}}$ promoter in response to blue

478 light instead of arabinose. BLADE TFs are compatible with previously constructed

479 strains carrying the $\mathrm{P}_{\mathrm{BAD}}$ promoter at an endogenous locus to drive the expression of a 480 gene of interest, allowing microbiologists to readily perform optogenetic experiments 481 without the need to construct anything new - transformation of the strain with pBLADE 482 is the only requirement. Moreover, since we constructed pBLADE using pBAD33 as 483 template (Supplementary Fig. 2a), microbiologists who wish to implement optogenetic 484 control of their gene of interest can simply re-clone it into pBLADE using the same restriction enzymes previously employed with pBAD33. Another advantage is that the resistance cassette and origin of replication of pBLADE are identical to those of pBAD33, thus ensuring compatibility with other previously constructed plasmids that

488 should be co-transformed with pBLADE. We additionally envisage that a plasmid 489 carrying BLADE under a constitutive promoter may be combined with previously constructed arabinose-inducible plasmids as long as the origin of replications and resistances are compatible. This strategy would require no cloning and guarantee full compatibility with established plasmids.

494 While many other light-inducible TFs have been developed to date $e^{61,68-72}$, some of 495 which featuring extremely high dark/light fold changes ${ }^{61,72}$, we explicitly aimed to engineer a system based on a well-known and pervasive TF (namely AraC) that is 497 particularly suited for microbiological applications, thus stimulating the use of 
optogenetics in microbiology. We took special care to engineer BLADE with minimal

499 leakiness. Often, leakiness has been assessed by comparing the levels of reporter 500 expression in the dark to those in the light. However, this does not take into account

501 whether the expression in the dark state is already too high compared to the expression

502 in the absence of the TF. Therefore, in the case of BLADE, minimal leakiness was

503 demonstrated by comparing its activity in the dark with expression obtained with the 504 same plasmid deprived of the TF (Fig.1f-i and Supplementary Fig. 2b). We put BLADE

505 to the test by expressing several functional E. coli proteins whose overexpression 506 causes morphological changes to the cells and showed that, in the dark, cells are 507 indistinguishable from the control (Fig. 3).

509 Light can be easily switched on and off. We showed that BLADE allows controlling a 510 phenotype in a fully reversible manner, using rod shape as an example (Fig. $3 g$, h). In 511 contrast to the fast reversibility achievable with pBLADE, the chemical inducer 512 arabinose, even after being washed off, remained inside the cells, committing them to 513 become even more rod-shaped instead of going back to the spherical morphology. 514 Moreover, light could be locally applied to create mixed populations to directly 515 compared the effects of morphology on the fitness of cells in different environments.

516 Another key feature of BLADE is that its leads to a homogenous response in a cell 517 population (Supplementary Fig. 3), in contrast to the heterogenous activation of $\mathrm{P}_{\mathrm{BAD}}{ }^{73}$.

518 While heterogeneity can be overcome by overexpression of either the arabinose 519 transporter $\mathrm{AraE}^{74}$ or $\mathrm{LacY}^{75}$, a transporter with relaxed specificity, the usage of the 520 promoter is then limited to these engineered strains or requires co-transformation with 521 a plasmid encoding the transporter. 
522 To showcase the utility of light induction in medium to high-throughput studies, we

523 used BLADE to overexpress 39 genes randomly selected from those with unknown or

524 poorly defined function. We applied four bioinformatic tools to predict their function

525 and localization. While it was possible to find a consensus prediction for $53 \%$ of the

526 genes in case of localization, only for $35 \%$ of the genes was a consensus found for

527 function prediction. Even in this case, the prediction remained relatively vague (ligase,

528 transporter, DNA binder, etc.; see Supplementary Table 1). This highlights how

529 computer-based predictions cannot yet replace detailed biochemical characterization,

530 which remains essential to assign a function to a protein.

531 Previous reports on light-inducible TFs based on VVD have employed untagged

532 versions, since the scope was to quantify the reporter gene expression output ${ }^{5,6,14,72}$.

533 We also did not include any FP in the BLADE construct at the beginning, since

534 visualization of BLADE localization was not important and the fusion may have not

535 been as active as the untagged version. It was, thus, surprising to see that BLADE is

536 not simply cytoplasmic as it may be expected (Fig. 5c). Formation of aggregates in the

537 dark is in good accordance with previous studies ${ }^{56,76}$. While we did not analyze VVD

538 alone, we speculate that the aggregates reflect a property of $\mathrm{VVD}$, not $\mathrm{AraC}_{\mathrm{DBD}}$.

539 Evidence in this respect comes from the results with BLADE bearing a mutated VVD

540 (VVD ${ }^{\mathrm{C} 108 \mathrm{~A}}$ ), which is insensitive to light and forms aggregates also under blue light

541 illumination (Fig. 5d). While in N. crassa VVD is degraded in the dark, in E. coli the

542 protein forms aggregates, which may effectively have an impact similar to degradation

543 in its rendering the protein inactive. The advantage of inactivation by sequestration

544 rather than degradation is that the protein can be quickly released from the aggregates

545 and activated when needed, without the delay that would result from a novel round of

546 gene expression. To date, many proteins have been shown to localize to intracellular 
547 bodies, however, often it is not clear if they constitute functional entities, such as liquid

548 droplets, storage bodies or aggregates ${ }^{77}$. For the protein stored in the aggregates to be

549 quickly activated, its association with these aggregates should be dynamic. When

550 followed over time in time-lapse microscopy, we found that the aggregates formed by

551 BLADE in the dark did not disperse in the same cell once it was illuminated with blue 552 light (Supplementary Video 2). The aggregates were polarly localized and 553 asymmetrically segregated to only one of the daughter cells (Supplementary Video 2).

554 This is in line with a previous report on asymmetric segregation of protein aggregates 555 in $E$. coli $^{78}$. These data suggest that the foci formed by BLADE are indeed 556 dysfunctional aggregates and that, when cells are illuminated, the probability of their 557 de novo formation strongly decreases. Notably, the aggregates cannot be the only 558 mechanism in place that controls the activation of the $\mathrm{P}_{\mathrm{BAD}}$ promoter by BLADE, since 559 we found them only in about half of the cells (Supplementary Fig. 9c). We believe they 560 add a layer of regulation to the system, contributing to its tightness. However, the 561 mechanism of BLADE-mediated gene expression involves dimer formation and 562 consequent occupancy of the $\mathrm{I}_{2}$ half-site, which recruits the RNA polymerase.

564 When engineering new TFs, we found that not only is an appropriate protein 565 engineering approach necessary, but also that the adjustment of the TF concentration is 566 critical if we wish to achieve optimal functionality. While this is intuitive for low TF 567 concentrations that might be insufficient to generate a biological response, we also 568 found that, if concentrations exceed certain levels, the functionality of the TF may 569 deteriorate (Fig. 6d) or disappear altogether (Fig. 6i). For the selected metric of 570 light/dark fold change, intermediate TF expression levels always led to the highest, and 571 hence the most optimal, values. Our high-throughput approach and novel 96-well light 
572 induction device made this possible. By calibrating the IPTG dose-response of an

573 inducible promoter to a large set of constitutive promoters, the desired TF expression

574 levels can be fixed, dispensing the need for an inducer (Fig. 6a). This mapping is

575 essential, because it dramatically reduces the experimental demand for cloning of

576 constructs, as only one construct needs to be cloned per TF. While this approach holds

577 great promise for the optimization and development optogenetic systems, as

578 demonstrated here, we expect that it will also be useful for the development of other

579 transcriptional regulators as well as for systematically bringing the expression levels of

580 various components in biological circuits to their optimal levels.

582 In this article we have demonstrated several features enabled by light induction, 583 including reversibility, which opens up the possibility of using dynamic inputs for 584 probing biological phenomena. In particular, pulsatile inputs that alternate between 585 dark (OFF) and maximum intensity (fully ON) can be used to achieve effects that 586 cannot be realized with graded intensity light. For example, it has been shown that 587 pulsatile inputs lead to reduced cell-to-cell variability in gene expression ${ }^{79}$. In fact, by 588 adjusting the duty-cycle (defined as the fraction of the time that the light is fully $\mathrm{ON}$ ), 589 one can even tune the amount of cell-to-cell variability, providing a new control 590 modality for exploring cell-to-cell variability and stochastic gene expression. This type 591 of pulsatile input was also recently shown to enhance the biosynthesis of products in 592 engineered cells, enabling a new manner of bioreactor operation in which enzyme expression is tuned to increase fermentation yield ${ }^{80}$. 
595 Taken together, the features of BLADE, its ease of adoption and usage, and its low

596 cost should bring the many benefits of optogenetic manipulation to the field of 597 microbiology, enabling new and exciting discoveries.

\section{Methods}

600 Strains, media and reagents. The strains used in this study are listed in Supplementary

601 Table 3. For experiments shown in Figures 1, 3-5, and Supplementary Figures 3, 4, 5 602 and 8, the cultures were grown in autoclaved Tryptone Broth (TB; $10 \mathrm{~g} \mathrm{l}^{-1}$ Tryptone, 5 $\left.603 \mathrm{~g} \mathrm{l}^{-1} \mathrm{NaCl}, 1 \mathrm{mM} \mathrm{NaOH}\right)$. For the bacterial photography experiments shown in Figure 6042 , and Supplementary Figure 7, the cultures were grown in autoclaved LB-Miller 605 medium. For experiments shown in Figure 6 and Supplementary Figures 12, 14-17, the 606 cultures were grown in autoclaved LB-Miller medium for strain propagation and in 607 sterile-filtered M9 medium supplemented with $0.2 \%$ casamino acids, $0.4 \%$ glucose, $6080.001 \%$ thiamine, $0.00006 \%$ ferric citrate, $0.1 \mathrm{mM}$ calcium chloride, $1 \mathrm{mM}$ magnesium 609 sulfate for all gene expression experiments. In experiments in which the plasmid had to 610 be maintained, the medium was supplemented with $34 \mu \mathrm{g} \mathrm{ml} \mathrm{m}^{-1}$ chloramphenicol 611 (Sigma-Aldrich Chemie GmbH). IPTG, rifampicin and doxycycline were purchased 612 from Sigma-Aldrich Chemie GmbH.

614 Constructions of strains and plasmids. To integrate $l a c Y A 177 C$ into the attB site of 615 BW25113 ${ }^{63,81}$ we used $\lambda$ integrase expressed from pJW27 using plasmid pSKA27 616 containing lacYA177C, FRT-flanked kanR from pKD13 ligated into XbaI-cut pFL503 ${ }^{82}$ 617 and a sequence identical to the genome regions for attB integration. pSKA27 was cut 618 with NotI, and the $4229 \mathrm{bp}$ band gel was purified and circularized before transformation 619 into pJW27-containing cells. For integration, pJW27 was transformed into E. coli 
BW25113 and selected at $30^{\circ} \mathrm{C}$ on LB-Agar plates containing chloramphenicol for

621 expression of $\lambda$ integrase. A single colony was used to inoculate $5 \mathrm{ml}$ of LB broth 622 containing chloramphenicol, and the culture was grown at $30^{\circ} \mathrm{C}$ in a water bath with 623 shaking. The cells were then moved to $42^{\circ} \mathrm{C}$ for $15 \mathrm{~min}$, before incubating on ice for $62415 \mathrm{~min}$. Cells were transformed with the integration construct using the previously 625 described transformation protocol. The fusion VVD-AraC proteins FP1-5 were first 626 subcloned into $\mathrm{pDK} 12^{83}$ using the NcoI and NotI restriction sites. The $v v d$ gene carrying 627 the N56K and C71V mutations and coding for a VVD protein missing the first 36 amino 628 acids was PCR-amplified out of plasmid pGAVPO (gift from Yi Yang; East China 629 University of Science and Technology). The araC fragments were amplified from 630 pBAD33. To clone the fusions, a two-step protocol was followed. In the first step, the 631 two parts were separately PCR-amplified. After the purification of the PCR products, 632 the two fragments were fused together in the second PCR step, and then cloned into 633 pDK12 with NcoI and NotI restriction enzymes, yielding plasmids pDK12(FP1-5). 634 Next, the DNA sequences coding for the fusion proteins FP1-FP5 were PCR-amplified 635 using primers 15 and 16 and cloned into pBAD33 (previously deprived of AraC via 636 PCR using primers 13 and 14, yielding the negative control plasmid pBLADE-empty) 637 linearized with ClaI. The J23101 promoter was included in the forward primer. The 638 obtained pBAD33-derived plasmids are called pBLADE(FP1-5). The $m$ Cherry gene 639 codon-optimized for expression in E. coli was synthesized (IDT) and cloned into 640 pBLADE with SacI and HindIII restriction enzymes. We subsequently generated 641 pBLADE(FP7)-mCherry by inserting an additional GS linker between VVD and AraC 642 with a site-directed mutagenesis protocol using pBLADE(FP4)-mCherry as template 643 with primers 17 and 18. The primers, designed with the QuikChange Primer Design 644 website, were used to amplify the plasmid. The PCR reactions contained $8 \%$ of DMSO 
645 to allow proper annealing of the primers to the template DNA. Similarly,

646 pBLADE(FP6)-mCherry was generated by removal of the GS linker from the same

647 template with the same protocol previously described, using primers 19 and 20 . To

648 construct the positive control with full-length AraC, pBLADE was linearized with ClaI,

649 the $\operatorname{araC}$ gene was PCR-amplified with primers 21 and 22 and then cloned into the 650 backbone, yielding pBLADE(AraC $\left.\mathrm{WT}_{\mathrm{WT}}\right)$-mCherry. The mutations and deletions leading 651 to promoters $\mathrm{J} 23101^{*}$ and J23101** (Supplementary Figure 1) generated 652 spontaneously during growth of bacterial cultures transformed with pBLADE(FP4)653 mCherry. These promoters have been subsequently cloned in all other pBLADE 654 plasmids by PCR-amplification with primers 23 and 24. The backbones were PCR 655 amplified with primers 25 and 26, yielding pBLADE(FP1*/FP7*)-mCherry and pBLADE(FP1**/FP7**)-mCherry. The promoters were cloned into pBLADE(AraC $\left.\mathrm{WT}_{\mathrm{T}}\right)$-mCherry by overlapping PCR starting from pBLADE(AraCwT)658 mCherry as template with primers 27 and 28, yielding $\mathrm{pBLADE}\left(\mathrm{AraC}_{\mathrm{WT}} * / \mathrm{AraC}_{\mathrm{WT}}^{* *}\right)$ 659 mCherry. The DNA sequence coding for eYFP-MinD was PCR-amplified out of pSR$66044^{84}$ with primers 31 and 32 and cloned into pBLADE(FP6*) via Gibson Assembly after 661 having amplified the backbone (pBLADE(FP6*)-mCherry) with primers 33 and 34.

662 The $s f g f p$ gene was PCR-amplified with primers 35 and 36 from plasmid pHR-scFv663 GCN4-sfGFP-GB1-NLS-dwPRE (gift from Ron Vale; Addgene plasmid \# 60906; 664 http://n2t.net/addgene:60906; RRID:Addgene_60906) and cloned in pBLADE(FP6*)665 mCherry via Gibson Assembly after amplification of the backbone with primers 37 and 666 38, yielding pBLADE(FP6*)-sfGFP. The DNA sequence coding for $\operatorname{MinD}^{\Delta 10}$ was 667 amplified out of pBDV-13 ${ }^{18}$ with primers 39 and 40 and cloned via Gibson Assembly 668 into pBLADE(FP4**)-mCherry previously amplified with primers 41 and 42 . The rodZ 669 and $m r e B$ genes were PCR-amplified from genomic DNA isolated from E. coli 
670 MG1655 using primers 43 and 44 and 45 and 46 respectively. Cloning into 671 pBLADE(FP4*) was achieved via Gibson Assembly after amplification of the 672 plasmid backbone with primers 33 and 34. pBAD\# (pBAD33 deprived of the $\mathrm{P}_{\mathrm{BAD}}$ 673 promoter and mCherry) was cloned via Gibson Assembly by amplification of 674 pBLADE(FP6**)-mCherry with primers 47 and 48, digestion of the linearized plasmid 675 with SacI and following ligation. The 39 genes with unknown or poorly defined 676 function were PCR-amplified from genomic DNA isolated from E. coli MG1655 using 677 the primer pairs listed in Supplementary Table 5. The backbone pBLADE(FP6**) was 678 amplified with primers 33 and 34 to insert the first 16 genes, and with primers 49 and 67950 to insert the others. These primers allow maintaining start and stop codon on the 680 plasmid backbone. To create the library with the fusion to sfGFP, the first 16 genes in 681 the list in Supplementary Table 1 were amplified with primers that included a GS linker 682 at their $\mathrm{N}$ or $\mathrm{C}$-terminus, and cloned in the backbone pBLADE(FP6**)-sfGFP 683 amplified with primers 33 and 51 (N-terminal fusions) and 52 and 34 (C-terminal 684 fusions). For the remaining genes, primers 50 and 54 (N-terminal fusions) and 49 and 68553 (C-terminal fusions) were used. For protein purification, the BLADE FP6 construct was PCR-amplified with primers 55 and 56 from pBLADE(FP6*)-mCherry and cloned 687 into pET28a with BamHI and NotI restriction enzymes, yielding to pET28a-FP6. In 688 order to invert the orientation of the $\mathrm{I}_{2}$ half-site, the plasmid pBLADE(FP6*)-mCherry 689 was amplified with overlapping PCR with primers 57 and 58 generating the plasmid 690 pBLADE_I $\mathrm{I}_{2}$ rev_(FP6*)-mCherry. Note that the -35 region, partially overlapping the $\mathrm{I}_{2}$ 691 half-site, was not inverted. For the fusion of sfGFP to the C-terminal of BLADE FP6, 692 pBLADE(FP6*)-mCherry was amplified with primers 59 and 60 , the $s f g f p$ gene was 693 amplified from pBLADE(FP6*)-sfGFP with primers 61 and 62, that carried a GS 694 linker. The cloning yielded to pBLADE((FP6-sfGFP)*)-mCherry. Gibson Assembly 
698 To clone the extended library of BLADE TFs, we used a modular Golden Gate cloning

699 strategy using an optimized junction set for part assembly taken from ${ }^{85}$. The overhangs 700 as well as the individual parts and the final plasmid sequences are shown in

701 Supplementary Table 6 as well as Supplementary Dataset 1 . To invert the 702 transcriptional unit containing the $m$ Cherry gene under AraC-controlled promoters, we 703 first assembled the transcriptional unit separately, and then PCR-amplified the resulting 704 fragment to create an A junction inverted at the end, and an $\mathrm{F}$ junction inverted at the 705 beginning of the transcriptional unit and further treated the resulting construct as a part. 706 Individual parts were first cloned into a part vector using BbsI-HF. The final plasmids 707 were assembled from individual parts with BsaI-HF for digestion of the parts, and BbsI$708 \mathrm{HF}$ for digestion of the plasmid backbone, which contains a p15a and a 709 chloramphenicol acetyl transferase. Plasmids were transformed using a one-step 710 preparation protocol of competent $E$. coli cells for transformation of plasmids in testing 711 strains ${ }^{86}$. The sequences of all cloned plasmids were confirmed by Sanger sequencing 712 (Eurofins Genomics Europe Sequencing GmbH, Köln, Germany, and Microsynth AG, 713 Balgach, Switzerland). A list of all vectors used and constructed in this study is shown 714 in Supplementary Table 4 and Supplementary Dataset 1. Oligonucleotide sequences 715 used for PCR amplification and Golden Gate part sequences are shown in 716 Supplementary Tables 5 and 6 . The cloning was performed using chemically competent 717 E. coli TOP10 cells (Thermo Scientific). 
Bacterial growth. For experiments shown in Figures 1-5 and Supplementary Figures

$\mathrm{g}, \mathrm{h}$ ) medium and grown at $37^{\circ} \mathrm{C}$ (with the exception of cultures used for the experiments in Figure $4 d$ and Supplementary Figure 8 which were grown at $18^{\circ} \mathrm{C}$ ) in an incubator shaking at $250 \mathrm{rpm}$, in black plastic tube (Argos Technologies LiteSafe ${ }^{\circledR}$ $15 \mathrm{ml}$ ) if containing light-sensitive samples, in transparent glass tubes otherwise (with the exception of cultures used for the experiments in Figure 4d and Supplementary Figure 8 which were shacked at $110 \mathrm{rpm}$ ). The following morning, the cultures were diluted to $\mathrm{OD}_{600} 0.1$ and let grow until $\mathrm{OD}_{600} 0.4$. Half of the culture was then transferred in transparent glass tubes and induced either with blue light or with arabinose for 4 hours (with the exception of cultures used for the experiments in Figure 4d and Supplementary Figure 8 which were diluted 1:30). For experiments shown in

732 Figure 6 and Supplementary Figures 12, and 14-17, cultures were grown in an 733 environmental shaker. The shaking incubator consisted of a Kuhner ES-X shaking 734 module (Adolf Kühner AG, Basel, Switzerland) mounted inside an aluminum housing 735 (Tecan, Maennedorf, Switzerland) and temperature-controlled using an "Icecube" (Life 736 imaging services, Basel, Switzerland). Cultures were grown at $37^{\circ} \mathrm{C}$ with shaking at $737300 \mathrm{rpm}$ in black, clear-bottom 96-well plates (Cell Culture Microplates 96 Well $738 \mu$ Clear ${ }^{\circledR}$ CELLSTAR ${ }^{\circledR}$, Greiner Bio-One GmbH, Product \#: 655090), which were 739 sealed with peelable foil (Sealing foil, clear peelable for PlateLoc, No. 16985-001, 740 Agilent) to prevent liquid evaporation and guarantee sterility, as well as a plastic lid 741 (Greiner Bio-One GmbH, Product \#: 656171). Overnight cultures were inoculated in 742 M9 medium and grown over night to an $\mathrm{OD}_{600}$ of about 4. These cultures were diluted $7431: 20,000$ into fresh M9 medium containing the respective inducer concentrations, right 
744 before the start of the experiment. This high dilution ensures that the cells are still in

745 logarithmic growth phase after $5 \mathrm{~h}$, at the end of the experiment ${ }^{61} .200 \mu \mathrm{l}$ of inoculated

746 culture were incubated per well in the 96-well plates. Cells were grown for $5 \mathrm{~h}$ before

747 transcription and translation was stopped with rifampicin and tetracycline ${ }^{61}$. The

748 inhibition solution contained $500 \mu \mathrm{g} \mathrm{ml}^{-1}$ rifampicin and $50 \mu \mathrm{g} \mathrm{ml}^{-1}$ tetracycline in

749 phosphate buffered saline (Sigma-Aldrich Chemie GmbH, Dulbecco's phosphate

750 buffered saline) and was filtered using a $0.2 \mu \mathrm{m}$ syringe filter (Sartorius). $100 \mu 1$

751 inhibition solution were aliquoted in 96-well U-bottom plates (Thermo Scientific

752 Nunc), precooled on ice and samples were added in equal volumes (100 $\mu 1)$, resulting

753 in a final inhibitor concentration of $250 \mu \mathrm{g} \mathrm{ml}^{-1}$ rifampicin (Sigma-Aldrich Chemie

$754 \mathrm{GmbH}$ ) and $25 \mu \mathrm{g} \mathrm{ml}^{-1}$ tetracycline (Sigma-Aldrich Chemie $\mathrm{GmbH}$ ). After sample was

755 added, the solution was incubated on ice for at least $30 \mathrm{~min}$. Then mCherry maturation

756 was carried out at $37{ }^{\circ} \mathrm{C}$ for $90 \mathrm{~min}$. The samples were kept at $4{ }^{\circ} \mathrm{C}$ until measurement

757 through flow cytometry.

758

759 Light illumination systems. To illuminate the glass tubes in the shaker, six high-power

760460 nm LEDs type CREE XP-E D5-15 (LED-TECH.DE) were used (Supplementary

761 Fig. 18). The LEDs were connected to a power supply (Manson HCS-3102) that

762 allowed to tune the voltage, hence the light intensity. Unless specified, the light

763 intensity reaching the cultures was $5 \mathrm{~W} / \mathrm{m}^{2}$ as measured with a LI-COR LI-250A Light

764 Meter. For the bacterial photography and the induction of the library of genes with

765 unknown or poorly defined function, we used a custom-made light box with, among

766 others, 6 blue (455 nm) LEDs (Supplementary Fig. 19). To avoid generation of a

767 blurred image in the bacteriograph, all the LEDs except for the one in the center were 
obscured with colored tape. The average light intensity reaching the plate was $5 \mathrm{~W} / \mathrm{m}^{2}$ with 6 LEDs and $1.3 \mathrm{~W} / \mathrm{m}^{2}$ with one LED.

The 96-LED array was designed using CircuitMaker 1.3.0 (www.circuitmaker.com).

771 The LEDs (SK6812, Dongguang Opsco Optoelectronics Co., Dongguan City, China)

772 were arranged on the PCB at a pitch of $9 \mathrm{~mm}$ in an $8 \times 12$ grid to be compatible with standard 96-well plates. All LEDs were daisy-chained using their DIN and DOUT ports. A $0.1 \mathrm{nF}$ capacitor was placed in parallel to the VDD port of each LED as

775 proposed by the manufacturer. The 2-layer circuit was manufactured on a $1.6 \mathrm{~mm}$ thick 776 FR-4 substrate, and the surface of the PCBs was coated with black solder mask to 777 reduce reflection. The PCBs were ordered preassembled with the LEDs and $0.1 \mathrm{nF}$ capacitors (www.pcbway.com, Shenzhen, China). Every 96-LED PCB had one signalin and one signal-out SMA connector such that several 96-LED PCBs could be daisychained using SMA cables and controlled by a single microcontroller. Up to 4x 96-

781 LED PCBs could be powered using a single Adafruit \#658 5V 10A switching power 782 supply (digikey.ch, Munich, Germany) using a custom-made PCB to distribute the 783 power to several LED arrays. The LEDs were controlled through an Arduino Uno 784 microcontroller (Arduino, Somerville, MA, USA) using the fastLED library 785 (http://fastled.io/).

786 The 96-LED array was mounted inside the shaking incubator using custom 3D-printed 787 holders. The holders were printed with an Ultimaker S5 using black Ultimaker CPE 788 (Ultimaker, Utrecht, Netherlands) to reduce reflections. For better dissipation and 789 distribution of the heat generated by the LEDs, a custom-made anodized aluminum 790 plate (10 mm thick, with 96 holes of 4 mm diameter) was mounted on top of the 96791 LED array. Another 3D-printed adapter was placed between the aluminum plate and 792 the microtiter plate to ensure optical insulation of the wells. The 3D-printed parts and 
793 the metal plate were aligned and held in place by metal rods $(4 \mathrm{~mm}$ diameter, $20 \mathrm{~mm}$

794 length).

795

796 Flow Cytometry. For experiments shown in Figures 1,3,4 and Supplementary Figures

$7973,4,5 b, 9 b$ and 9c, fluorescence was measured using the LSR Fortessa flow cytometer

798 (BD Biosciences). Samples were centrifuged at $4000 \mathrm{~g}$ for $4 \mathrm{~min}$ to remove the glycerol-

799 containing solution, then the pellets were resuspended in PBS. Data analysis was

800 performed using the open source FCSalyzer software. The mCherry fluorescence was

801 excited with a $561 \mathrm{~nm}$ laser $(50 \mathrm{~mW})$, and emission was detected using a 610/20-nm

802 filter pass (PMT voltage set to $750 \mathrm{~V}$ ). The GFP fluorescence was excited with $488 \mathrm{~nm}$

803 laser (100 mW), and emission was detected using a 530/30-nm filter pass (PMT voltage

804 set to $405 \mathrm{~V}$ ). A forward scatter height (FSC-H) threshold of 1,400 was used to gate for

805 living cells and eliminate debris. $10^{5}$ events per sample were recorded for each

806 experiment. The cell density of the samples was manually regulated by addition of PBS

807 in order to have less than $2 * 10^{4}$ events/s recorded by the machine. To compensate any

808 variable that can alter the measurement of the fluorescence by the flow cytometer, each

809 experiment was normalized with the fluorescence value of the negative control grown

810 the same day of the experiment. For experiments shown in Figure 6 and Supplementary

811 Figures 11, 12, 14, 15-17, fluorescence was measured on a Cytoflex S flow cytometer

812 (Beckman Coulter) equipped with CytExpert 2.1.092 software. The mCherry

813 fluorescence was excited with a $561 \mathrm{~nm}$ laser and emission was detected using a 610/20

$814 \mathrm{~nm}$ band pass filter and following gain settings: forward scatter 100, side scatter 100,

815 mCherry gain 3,000 when mCherry was expressed from the $\mathrm{I}_{1}-\mathrm{I}_{2}$ promoter, and 300

816 gain when mCherry was expressed from the $\mathrm{I}_{1}-\mathrm{I}_{1}$ promoter due to the difference in

817 expression levels. Thresholds of 2,500 FSC-H and 1,000 SSC-H were used for all 
818

819

samples. The flow cytometer was calibrated before each experiment with QC beads (CytoFLEX Daily QC Fluorospheres, Beckman Coulter) to ensure comparable fluorescence values across experiments from different days. At least 15,000 events or 2 min were recorded in a two-dimensional forward and side scatter gate, which was drawn by eye and corresponded to the experimentally determined size of the testing strain at logarithmic growth and was kept constant for analysis of all experiments and used for calculations of the median and CV using the CytExpert software. The same gating strategy was previously used and is depicted in Supplementary Figure 21.

Characterization of the FP1-FP7 VVD-AraC ${ }_{\text {DBD }}$ fusion constructs. Chemically competent E. coli MG1655 cells were transformed with pBLADE(FP1*/FP7*)mCherry, pBLADE(FP1**/FP7**)-mCherry, pBLADE-empty (negative control), and pBLADE(AraC $\left.\mathrm{WT}^{*} / \mathrm{AraC}_{\mathrm{WT}}{ }^{* *}\right)$-mCherry (positive controls). Overnight cultures of cells transformed with the FP1-FP7 fusions were diluted to $\mathrm{OD}_{600} 0.1$, let grow in the dark to $\mathrm{OD}_{600} 0.4$ and split into two cultures, one of which was kept in the dark and one of which was illuminated for $4 \mathrm{~h}$. The overnight culture of the negative control was diluted to $\mathrm{OD}_{600} 0.1$, and let grow for the same amount of time as all other cultures (circa $5 \mathrm{~h} 30 \mathrm{~min}$ ). The overnight cultures of the positive controls were diluted to $\mathrm{OD}_{600}$ 0.1 , let grow to $\mathrm{OD}_{600} 0.4$ and split into two cultures, one of which was left without arabinose and one of which was induced with $0.1 \%$ arabinose for $4 \mathrm{~h}$. After the induction time, $200 \mu 1$ of each sample were collected, mixed with $200 \mu$ of a transcription and translation inhibition solution $\left(500 \mu \mathrm{g} \mathrm{ml}^{-1}\right.$ rifampicin and $50 \mu \mathrm{g} \mathrm{ml}^{-1}$ doxycycline in phosphate buffered saline) and incubated in the dark $90 \mathrm{~min}$ at $37^{\circ} \mathrm{C}$ with $110 \mathrm{rpm}$ shaking. This protocol allows obtaining a full maturation of almost all the mCherry proteins translated at the end of the induction time ${ }^{1}$. After the incubation 
843

844

845

846

847

848

849

850

851

852

853

854

855

856

857

858

859

860

861

862

863

864

865

866 Light intensity titration. Chemically competent E. coli MG1655 cells were

with the inhibitor, samples were either diluted 10 times with PBS and immediately analyzed with the flow cytometer, or diluted $1: 1$ with $60 \%$ glycerol and frozen at $-80^{\circ} \mathrm{C}$.

Dynamic control of gene expression. The overnight cultures transformed with pBLADE(FP6**)-mCherry and pBLADE-empty (negative control) were diluted in TB to $\mathrm{OD}_{600} 0.05$ in dark tubes and let grow until $\mathrm{OD}_{600}$ 0.15. $200 \mu \mathrm{l}$ of each sample were collected, mixed with $200 \mu 1$ of a transcription and translation inhibition solution (500 $\mu \mathrm{g} \mathrm{ml} \mathrm{f}^{-1}$ rifampicin and $50 \mu \mathrm{g} \mathrm{ml}^{-1}$ doxycycline in phosphate buffered saline), incubated in the dark 90 min at $37^{\circ} \mathrm{C}$ with $110 \mathrm{rpm}$ shaking, diluted $1: 1$ with $60 \%$ glycerol, and frozen at $-80^{\circ} \mathrm{C}$. The rest of the culture was transferred in a transparent glass tube and illuminated with blue light as described (Light illumination systems) for $2 \mathrm{~h}$. Then, another aliquot was taken and frozen, and the remaining culture was diluted to $\mathrm{OD}_{600}$ 0.15 again and transferred to a dark tube, for a total of three dark-light cycles.

Measurement of the kinetics of BLADE-mediated mCherry expression. Chemically competent E. coli MG1655 cells were transformed with pBLADE(FP6*)mCherry and pBLADE-empty. The overnight cultures were diluted and each split into two cultures, of which one was induced with blue light and one kept in the dark. Every hour for $6 \mathrm{~h}, 200 \mu \mathrm{l}$ of each sample were collected, mixed with $200 \mu \mathrm{l}$ of the transcription and translation inhibition solution, incubated in the dark $90 \mathrm{~min}$ at $37^{\circ} \mathrm{C}$ with $110 \mathrm{rpm}$ shaking, diluted $1: 1$ with $60 \%$ glycerol, frozen at $-80^{\circ} \mathrm{C}$ and subsequently analyzed with the flow cytometer.

transformed with pBLADE(FP6**)-mCherry and pBLADE-empty. The overnight 
culture of the cells transformed with pBLADE(FP6**)-mCherry was diluted and split into 5 independent cultures, each of which was induced with blue light of different intensity (which was tuned adjusting the voltage in the power supply connected to the LEDs) for $4 \mathrm{~h}$. The overnight culture of the cells transformed with pBLADE-empty was diluted and grown in the dark for $4 \mathrm{~h} .200 \mu \mathrm{l}$ of each sample were then collected, mixed with $200 \mu 1$ of the transcription and translation inhibition solution, incubated in the dark 90 min at $37^{\circ} \mathrm{C}$ with $110 \mathrm{rpm}$ shaking, diluted $1: 1$ with $60 \%$ glycerol, frozen at $-80^{\circ} \mathrm{C}$ and subsequently analyzed with the flow cytometer.

Bacterial photography. Chemically competent E. coli MG1655 cells were transformed with pBLADE(FP6*)-sfGFP. The overnight culture was diluted in LB to $\mathrm{OD}_{600} 0.1$ and grown for approximately $6 \mathrm{~h}$. A 96-well lid $(12.7 \times 8.5 \mathrm{~cm})$ was filled with $30-40 \mathrm{ml}$ of $1 \%$ LB-agar and let solidify. $1 \mathrm{ml}$ of the culture was then mixed with $9 \mathrm{ml}$ of $0.4 \%$ agar at $42^{\circ} \mathrm{C}$ (measured with infrared thermometer TFA Dostmann (Wertheim-Reicholzheim, Germany)) and plated on top of the solidified agar in the 96well lid. The plate was covered with a transparent plexiglass parallelepiped with the Blade Runner movie poster sticker. To increase the opacity of the dark zones of the picture, three identical stickers were overlapped on one another. The plate was then placed in a $37^{\circ} \mathrm{C}$ incubator under the light box overnight. The next morning the plate was imaged with a Zeiss Axio Zoom.V16 stereo zoom microscope equipped with PlanNeoFluar Z 1.0x objective, zoom 0.7x, AxioCam MR R3 camera and the $38 \mathrm{HE}$ filter set (Ex BP 470/40, FT 495, Em BP 525/50; sfGFP). The bacteriograph is composed of 110 tiles stitched together with ZEN Blue software. 
DIC and fluorescence microscopy. $5 \mu 1$ of the bacterial culture were applied to a thin agarose pad composed of $1 \%$ agarose for microscopy at room temperature and of $1 \%$ agarose and $0.1 \%$ LB in Tethering buffer $(10 \mathrm{mM}$ potassium phosphate, $0.1 \mathrm{mM}$ EDTA, $1 \mathrm{mM}$ L-methionine and $10 \mathrm{mM}$ sodium lactate; $\mathrm{pH} 7.0$ ) for long-term microscopy at $37^{\circ} \mathrm{C}$. Images were acquired on a Zeiss Axio Observer Z1/7 fluorescence microscope equipped with an Alpha Plan-Apochromat 100x/1.46 Oil DIC (UV) M27 objective, filter sets $38 \mathrm{HE}$ (Ex BP 470/40, FT 495, Em BP 525/50; sfGFP), $108 \mathrm{HE}$ (Ex BP 423/44, DBS 450+538, Em DBP 467/24+598/110; MM 4-64), 96 HE (Ex BP 390/40, FT 420, Em BP 450/40; DAPI), 64 HE (Ex BP 587/25, FT 605, Em BP 647/70;

901 mCherry) and an Axiocam 506 Mono camera. To image the library of genes with unknown or poorly defined function in a fast and efficient way, the samples (circa 5 ml) were applied to a 96-well lid, which was filled with $1 \%$ agarose, let solidify and 904 covered with two 75 x $50 \mathrm{~mm}$ glass coverslips (Carl Roth GmbH, Karlsruhe). Before 905 imaging, samples were incubated for 5 min with $1.2 \mu \mathrm{g} \mathrm{ml}^{-1}$ of the membrane dye MM 4-64 (AAT Bioquest Sunnyvale, CA) and $0.5 \mu \mathrm{g} \mathrm{ml}^{-1}$ of 4',6-diamidino-2-phenylindole (DAPI, Sigma-Aldrich Chemie GmbH).

908 The induction of gene expression in selected cells within a population of MG1655 cells 909 transformed with pBLADE(FP6**)-sfGFP was performed on a Zeiss LSM 800 910 confocal microscope. An area of $6.4 \mu \mathrm{m}^{2}$ was illuminated with a $488 \mathrm{~nm}$ diode laser $911(10 \mathrm{~mW})$ at $0.1 \%$ intensity, with a frame average of 8 , resulting in $0.36 \mu$ s of light per 912 pixel. The illumination was given in pulses of $5 \mathrm{~min}$ for a duration of $3 \mathrm{~h}$.

914 FRAP. FRAP was performed on a Zeiss LSM 800 confocal microscope. An overnight 915 culture of MG1655 cells transformed with pBLADE((FP6-sfGFP)*)-mCherry was 916 diluted in the morning in fresh TB medium to $\mathrm{OD}_{600} 0.1$, and grown until it reached 
$917 \mathrm{OD}_{600}$ 0.4. $5 \mu$ l of the culture were then applied to a thin $1 \%$ agarose pad. After selecting

918 a cell with a bright fluorescent spot, the area to bleach within the cell (whole spot) was

919 manually set (the whole focus) and bleached with a single $1 \mathrm{~s}$ pulse of a $488 \mathrm{~nm}$ diode

920 laser (10 mw) at 50\% intensity. An image in the GFP channel (filter set $38 \mathrm{HE}$ : Ex BP

$921470 / 40$, FT 495, Em BP 525/50) was taken 5- and 15-min post bleaching to measure

922 the recovery of the fluorescent signal.

923

924 Induction of rodZ in KC717 cells. Strain KC717 (kind gift of KC Huang, Stanford

925 University) was grown in LB medium supplemented with $0.2 \%$ arabinose (to maintain

926 the cells rod-shaped) during transformation of chemically competent KC717 cells and

927 DNA extraction procedures. The blue light and arabinose induction were performed as

928 described above. The recovery phase of the culture induced with arabinose was

929 performed by centrifuging it at $6000 \mathrm{~g}$ for $4 \mathrm{~min}$ and resuspending it with the same

930 volume of LB. The centrifugation and resuspension steps were repeated a second time

931 and the culture was then diluted to $\mathrm{OD}_{600} 0.1$. The recovery phase of the culture 932 transformed with pBLADE(FP4**) was performed by dilution of the culture exposed 933 to blue light $\mathrm{OD}_{600} 0.1$ and incubation in the dark.

935 BLADE (FP6) expression and purification. Chemically competent E. coli Rosetta

936 (DE3) cells carrying the pLysS plasmid were freshly transformed with pET28a-FP6

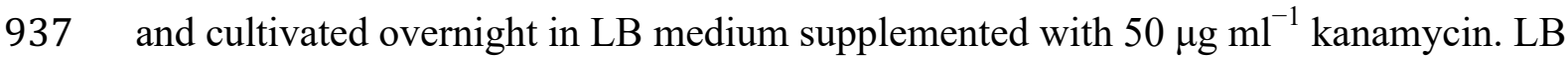

938 medium (1 1 l) containing kanamycin was inoculated using the pre-culture to obtain

$939 \mathrm{OD}_{600}$ of 0.1 . The culture was grown at $37^{\circ} \mathrm{C}$ until $\mathrm{OD}_{600}$ of 0.5 , after which $1 \mathrm{mM}$

940 IPTG and $5 \mu \mathrm{M}$ FAD were added, and the culture was grown for $16 \mathrm{~h}$ at $18^{\circ} \mathrm{C}$ under

941 constant blue light. Cells were collected by centrifugation and the pellet was re- 
942

943

944

945 medium) was subtracted from the dark and lit state spectra. absorption spectrum (Supplementary Fig. 20).

suspended in $30 \mathrm{ml}$ of lysis buffer $(50 \mathrm{mM}$ potassium phosphate $\mathrm{pH} 8.0,300 \mathrm{mM} \mathrm{NaCl}$ and $10 \mathrm{mM}$ imidazole $\mathrm{pH}$ 8.0) supplemented with a cOmplete ${ }^{\mathrm{TM}}$ protease inhibitor cocktail tablet (Roche). Cell lysis was performed by sonication and the lysate was centrifuged at $20,000 \mathrm{rpm}$ for $20 \mathrm{~min}$ at $4^{\circ} \mathrm{C}$. The supernatant was then co-incubated with $1 \mathrm{ml}$ of HisPur ${ }^{\mathrm{TM}} \mathrm{Ni}-\mathrm{NTA}$ Resin (Thermo Scientific) for $2 \mathrm{~h}$ at $4^{\circ} \mathrm{C}$. Protein purification was performed by the gravity flow method. The bound proteins were washed twice with $5 \mathrm{ml}$ of wash buffer (lysis buffer $+10 \%$ glycerol $+20 \mathrm{mM}$ imidazole) and finally eluted with $1.5 \mathrm{ml}$ of elution buffer $(50 \mathrm{mM}$ potassium phosphate pH 7.5, $300 \mathrm{mM} \mathrm{NaCl}, 500 \mathrm{mM}$ imidazole $\mathrm{pH} 8.0$ and $10 \%$ glycerol). The elution buffer was replaced with a storage buffer $(20 \mathrm{mM}$ HEPES-NaOH pH 7.5, $150 \mathrm{mM}$ $\mathrm{NaCl}$ and $10 \%$ glycerol) using an Amicon ${ }^{\circledR}$ Ultra-4 regenerated cellulose NMWL 10 $\mathrm{kDa}$ centrifugal filter unit (Merck). The protein was then stored as $50 \mu \mathrm{l}$ aliquots at $80^{\circ} \mathrm{C}$. We verified that the purified protein could respond to light by measuring the

Spectroscopy. The absorption spectrum of the FAD cofactor bound to VVD within BLADE (FP6) was measured exciting the sample in the 300-600 $\mathrm{nm}$ range using a Multiskan GO (Thermo Scientific) plate reader. The protein sample was incubated 4 days at $4{ }^{\circ} \mathrm{C}$ in the dark in a buffer solution (25 mM HEPES, $150 \mathrm{mM} \mathrm{NaCl}, 10 \%$ glycerol, $0.1 \%$ EDTA; $\mathrm{pH} 7.5$ ) and then diluted to $0.5 \mathrm{mg} \mathrm{ml}^{-1}$ prior to the measurement of the absorption spectrum in the dark state. The same sample was then illuminated with blue light $\left(455 \mathrm{~nm} ; 50 \mathrm{~W} / \mathrm{m}^{2}\right)$ for 5 min at room temperature and the absorption spectrum in the lit state was measured. The absorption spectrum of the blank (only 
SEC. Purified BLADE (FP6) was thawed and stored in complete darkness at $4^{\circ} \mathrm{C}$ for 6 running buffer consisted of $20 \mathrm{mM}$ HEPES-NaOH pH 7.5, $150 \mathrm{mM} \mathrm{NaCl}$ and $10 \%$

971 glycerol, and the flowrate was adjusted to $0.25 \mathrm{ml} \mathrm{min}^{-1}$. Dimerization of BLADE FP6 was triggered by incubating the protein under constant blue light $\left(455 \mathrm{~nm} ; 50 \mathrm{~W} / \mathrm{m}^{2}\right)$

975 (dark sample). Bovine serum albumin (BSA) and carbonic anhydrase (CA) were used as size markers at a concentration of $0.5 \mathrm{mg} \mathrm{ml}^{-1}$ each.

Light-induced expression of genes with unknown or poorly defined function.

979 Chemically competent MG1655 cells were transformed with the 117 pBLADE 980 plasmids constituting the library to characterize the 39 genes with unknown or poorly 981 defined function. Cultures were grown in the dark overnight in LB in non-treated 96well plate (VWR, Radnor, PA) at $37^{\circ} \mathrm{C}$ with $110 \mathrm{rpm}$ shaking. The following morning transfer about $5 \mu$ l of each culture into a fresh 96-well plate with $145 \mu 1$ of TB in each

985 well. The diluted cultures were incubated at $18^{\circ} \mathrm{C}$ with $110 \mathrm{rpm}$ shaking for $1 \mathrm{~h}$ in the 986 dark and then they were induced with blue light $\left(455 \mathrm{~nm}, 5 \mathrm{~W} / \mathrm{m}^{2}\right)$ for $4 \mathrm{~h}$.

988 Measurement of bacterial growth. The growth curves of the cells transformed with 989 the library of genes with unknown or poorly defined function were measured on a 990 Synergy H4 Hybrid plate reader (BioTek) in 96-well plates. The cultures were grown 991 in the dark overnight in LB in a $96-$ well plate at $37^{\circ} \mathrm{C}$ with $110 \mathrm{rpm}$ shaking. The 
following morning the cultures were diluted to $\mathrm{OD}_{600} 0.1$ in a fresh 96 -well plate with

$993120 \mu \mathrm{l}$ of LB. To prevent evaporation of the medium, also the unused wells of the plate

994 were filled with the same amount of LB and the lid was sealed with parafilm. The plate 995 was then illuminated with blue light $(460 \mathrm{~nm})$ and the $\mathrm{OD}_{600}$ of the culture was 996 measured every 2 min in constant shaking for $20 \mathrm{~h}$.

997 The overnight cultures of three selected members of the library (ydaT, $y d h L, y h c F$ ) 998 were diluted in $\mathrm{LB}$ to $\mathrm{OD}_{600} 0.1$ and grown until they reached $\mathrm{OD}_{600} 0.4$. Each culture 999 was then split into two tubes, one of which was kept in the dark and one of which was 1000 illuminated for $4 \mathrm{~h}$ at $37^{\circ} \mathrm{C}$ with shaking at $250 \mathrm{rpm}$. The $\mathrm{OD}_{600}$ was measured at the 1001 end of the growth with the OD600 DiluPhotometer ${ }^{\mathrm{TM}}$ (Implen).

Quantification of cell length, width and roundness. The cell length and width were

1004 calculated by first staining the cell with the membrane dye MM 4-64 (AAT Bioquest 1005 Sunnyvale, CA) to visualize the cell contour, and then manually measuring the long 1006 and short axes of the cell, respectively, using the straight-line 'Selection' tool of Fiji. 1007 At least 500 cells were measured for each sample. The histograms were generated in 1008 Excel by the Analysis ToolPak's Histogram option. The roundness was manually 1009 calculated with the oval 'Selection' tool on unstained cells, using Fiji. At least 200 cells 1010 were measured for each sample.

1012 Computational prediction of function and localization of $\mathbf{3 9}$ genes with unknown 1013 or poorly defined function. We randomly selected 34 genes out of the y-ome, defined 1014 as the group of genes lacking to date experimental evidence of function ${ }^{36}$. We manually 1015 checked that the selected genes were not mentioned in any publication using several 1016 search engines. As controls for our pipeline, we included 5 genes for which some 
1017 information was available $\left(y d a T^{37} ; y d i Y^{38} ; y c b K(\mathrm{MepK})^{39} ; y e h S^{40}\right.$; and $\left.y e b E^{41,42}\right)$. We

1018 retrieved the amino acid sequences of the proteins encoded by all 39 genes in FASTA

1019 format and submitted them to the following webservers: Argot2.546, PANNZER2 ${ }^{47}$,

1020 DeepGoPlus ${ }^{48}$ and Phyre $2^{49}$. The consensus localization and function were calculated 1021 as the output provided by at least $2 / 4$ prediction tools.

1023 Mathematical modelling. The LacI IPTG dose-response was fitted to a Hill equation 1024 of the following form:

$$
f(x)=r_{\max } \frac{x^{n}}{k_{\mathrm{m}}+x^{n}}
$$

1026 where $f(x)$ describes the gene expression controlled by LacI, $x$ represents the IPTG 1027 concentration, $r_{\max }$ is the maximal promoter expression, $k_{m}$ is IPTG's dissociation 1028 constant for LacI, and $n$ is the Hill coefficient for LacI. This dose-response was used 1029 subsequently to obtain IPTG concentration estimates from the fluorescence readouts of 1030 the constitutive promoters. All data were fitted using a non-linear least squares 1031 optimizer (MATLAB, MathWorks) with fitted parameter values $r_{\max }=21352, \mathrm{k}_{\mathrm{m}}=62$, $1032 \mathrm{n}=1.7$

\section{Acknowledgements}

1035 We thank Maximilian Hörner for his help with the determination of the absorption 1036 spectrum of BLADE, João Nuno de Sousa Machado for his help with size-exclusion 1037 chromatography, Yanik Weber for help with characterizing the 96-well light induction 1038 plate, KC Huang for sharing with us KC717 strain, and Stephanie Aoki for helpful 1039 discussions. This study was funded by the DFG (grant no. VE776/2-1 to B.D.V.), by 1040 the BMBF (grant no. 031L0079 to B.D.V.), by the Excellence Initiative of the German 1041 Federal and State Governments BIOSS (Centre for Biological Signalling Studies; EXC- 

294), by the European Research Council (ERC-Advanced) under the European Union's Horizon 2020 research and innovation programme (grant agreement number 743269).

\section{Author contributions}

B.D.V. and A.B. conceived the study. B.D.V and M.K. supervised the study, and secured funding. E.R., A.B., E.A., N.P., M.K. and B.D.V. designed experiments and interpreted the data. E.R., A.B., M.H. and E.A. performed in vivo experiments. N.P. purified BLADE, and performed size-exclusion chromatography. L.E. performed initial experiments, which validated the idea. G.S. developed the 96-well light setup in collaboration with A.B. M.A.Ö. performed bioinformatics and computational structural biology analyses of the genes with unknown function. E.R., A.B., M.K. and B.D.V. wrote the manuscript.

\section{Competing interests}

The authors declare no competing interests.

\section{Data availability}

The plasmids constructed in this study will be deposited on Addgene and will be additionally available from the corresponding authors upon request. The raw data supporting the conclusions of the paper will also be available from the corresponding authors upon request.

(1) 
Figures

a
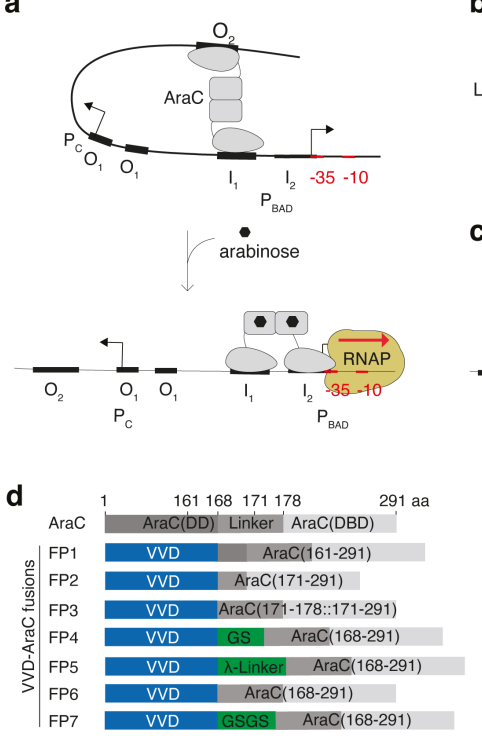

f

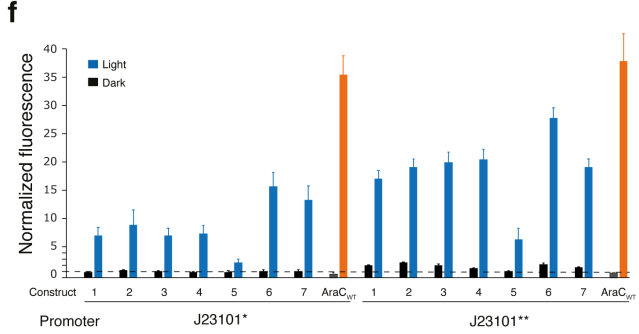

h

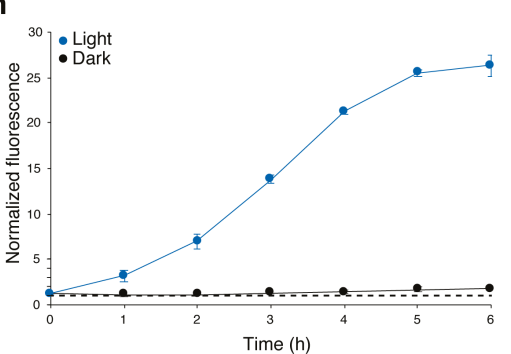

b Native AraC protein Light-inducible AraC

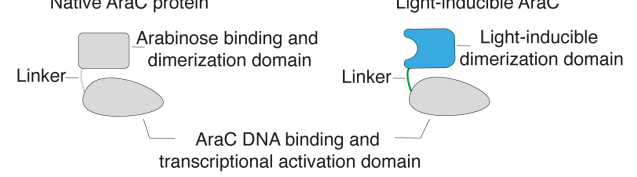

C

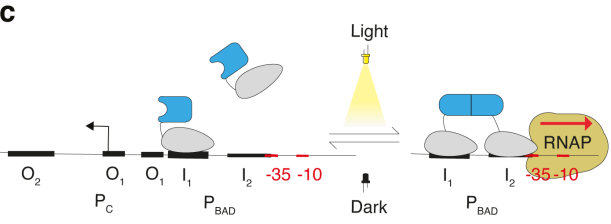

e

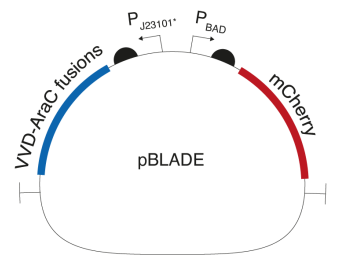

g

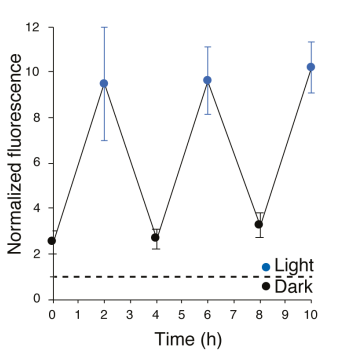

i

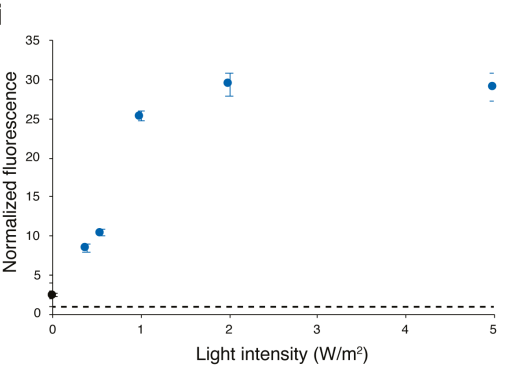

Fig. 1| Engineering and characterization of a novel light-inducible AraC a, Mechanism of arabinoseinduced $\mathrm{P}_{\mathrm{BAD}}$ induction by AraC. The thickness of the $\mathrm{I}_{1}$ and $\mathrm{I}_{2}$ half-sites is proportional to the affinity with which $\mathrm{AraC}$ binds to them. $\mathrm{P}_{\mathrm{C}}$, promoter driving the expression of $\operatorname{araC}$. $\mathbf{b}$, Domain composition of wild type (left) and light-inducible (right) AraC. c, Expected mechanism of $\mathrm{P}_{\mathrm{BAD}}$ activation by lightinducible AraC. d, Domain composition of the chimeric VVD-AraC $C_{D B D}$ fusion constructs. In FP3, amino acids 171-178 of the natural linker are present twice. e, Plasmid for expression of a gene of interest (here mCherry) under control of BLADE. f, mCherry fluorescence intensity in E. coli MG1655 cells transformed with the library shown in (d) grown for $4 \mathrm{~h}$ either in the dark or under $460 \mathrm{~nm}$ light $\left(5 \mathrm{~W} / \mathrm{m}^{2}\right)$ illumination. Native AraC cloned under the same constitutive promoters was used as positive control. The bars for $\mathrm{AraC}$ represent the values obtained without (black) and with $0.1 \%$ (orange) arabinose for 4h. g, mCherry fluorescence intensity in the same culture of E. coli MG1655 cells transformed with the FP6 fusion driven by the $\mathrm{J} 23101^{* *}$ promoter after repeated cycles of blue light exposure and darkness. $\mathbf{h}$, Kinetics of mCherry expression in E. coli MG1655 cells transformed with the FP6 fusion driven by the J23101* promoter grown for 4 h either in the dark or under $460 \mathrm{~nm}$ light $\left(5 \mathrm{~W} / \mathrm{m}^{2}\right)$ illumination. $\mathbf{i}$, mCherry fluorescence intensity measured in E. coli MG1655 cells transformed with the FP6 fusion driven by the $\mathrm{J} 23101^{* *}$ promoter grown for $4 \mathrm{~h}$ under $460 \mathrm{~nm}$ light of the indicated light intensity (cyan) or kept in the dark for $4 \mathrm{~h}$ (black). f-i, All values were normalized to the mCherry fluorescence intensity measured in E. coli MG1655 cells transformed with the plasmid deprived of the transcription factor (dashed line). Values represent mean \pm s.d. of at least $n=3$ independent experiments. 
bioRxiv preprint doi: https://doi.org/10.1101/2020.07.14.202911; this version posted July 14, 2020. The copyright holder for this preprint (which was not certified by peer review) is the author/funder, who has granted bioRxiv a license to display the preprint in perpetuity. It is made available under aCC-BY-NC-ND 4.0 International license.

b

a

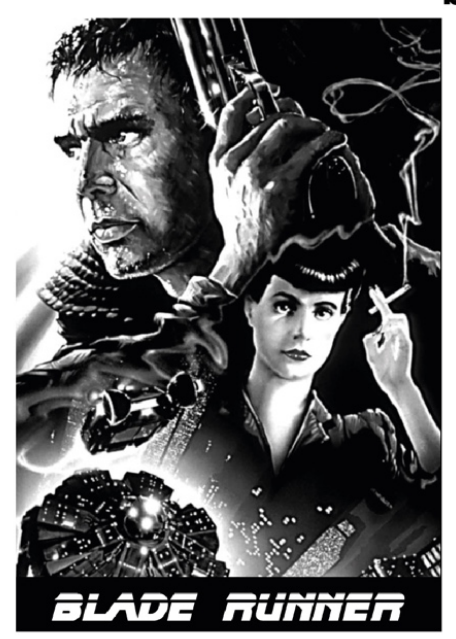

C
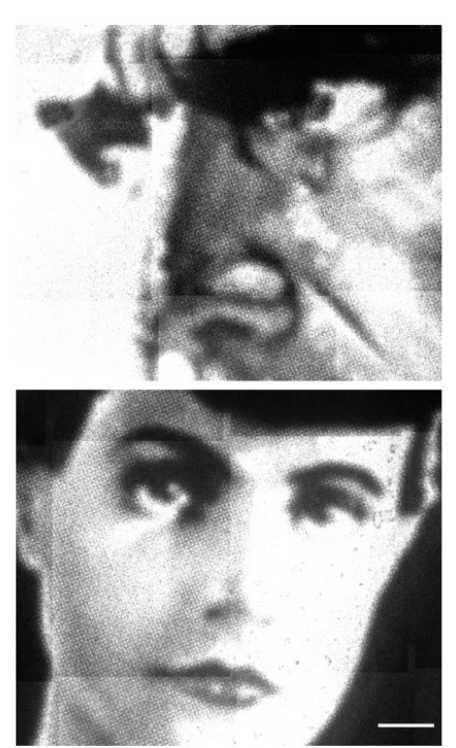

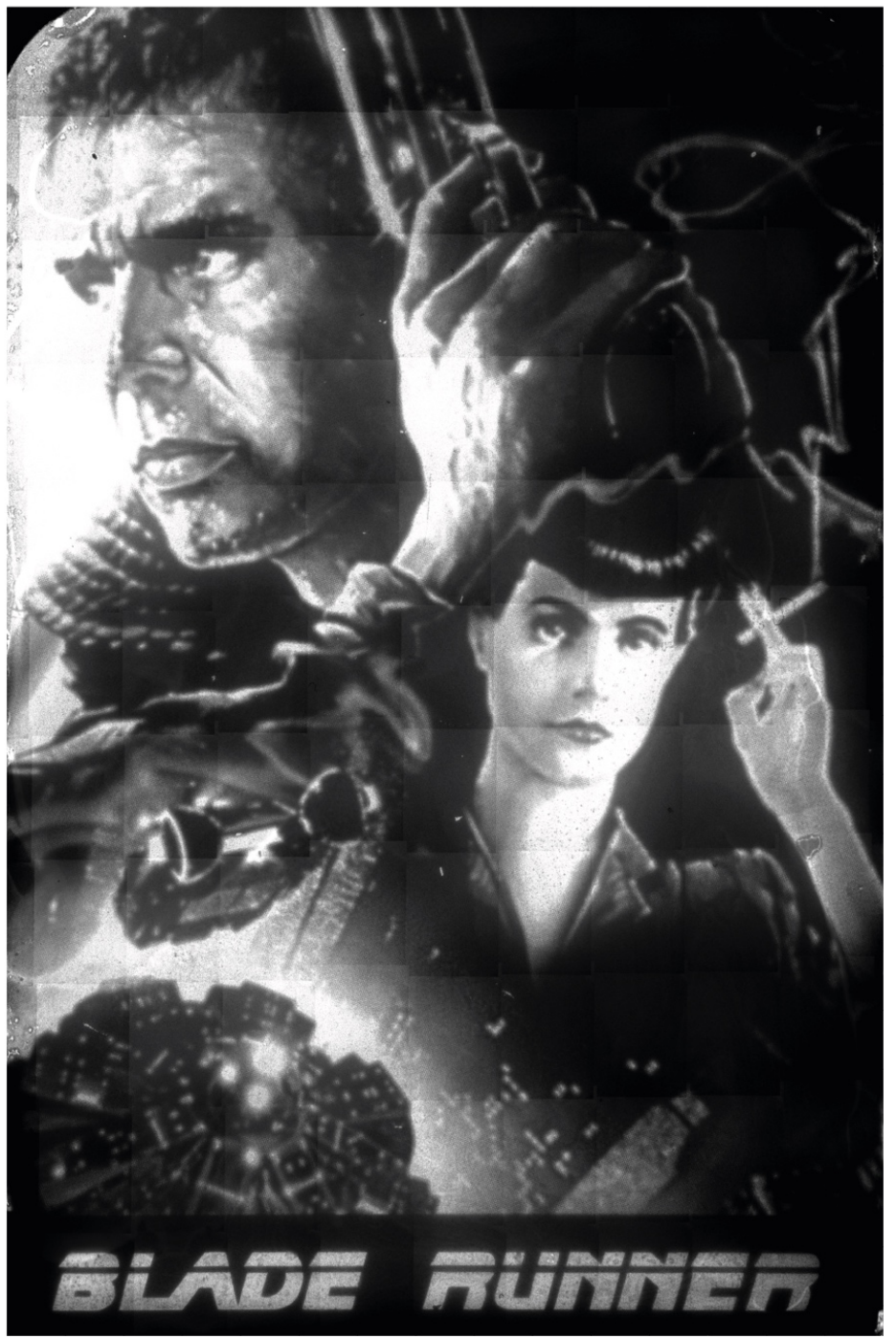

Fig. 2| BLADE allows for the production of high contrast bacteriographs. a, Photomask used to produce the bacteriograph (printed with permission from Warner Bros. Entertainment, Inc.). b, Bacteriograph. A lawn of $E$. coli MG1655 cells transformed with pBLADE(FP6*)-sfGFP were grown overnight at $37^{\circ} \mathrm{C}$ while being exposed to blue light through the photosmask in (a). 110 individual images were taken with a fluorescent microscope and stitched together via image analysis software. Scale bar, 1 $\mathrm{cm}$. $\mathbf{c}$, Zoom in on two parts of the bacteriographs. Scale bar, $300 \mu \mathrm{m}$. 
a

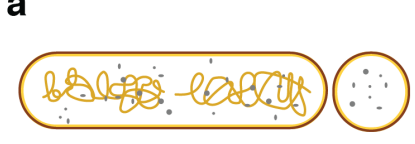

Mini-cell phenotype

b

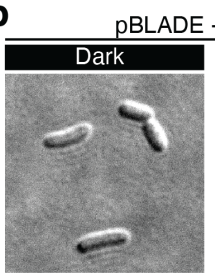

c

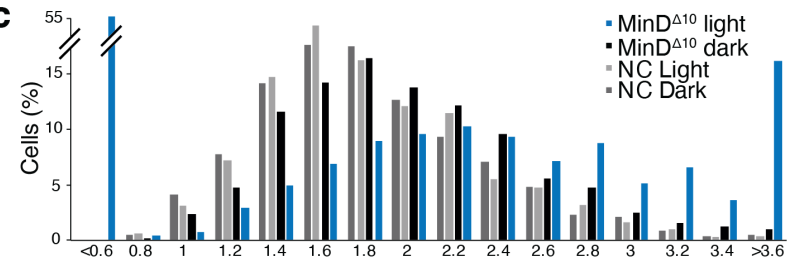

E. coli length $(\mu \mathrm{m})$

e
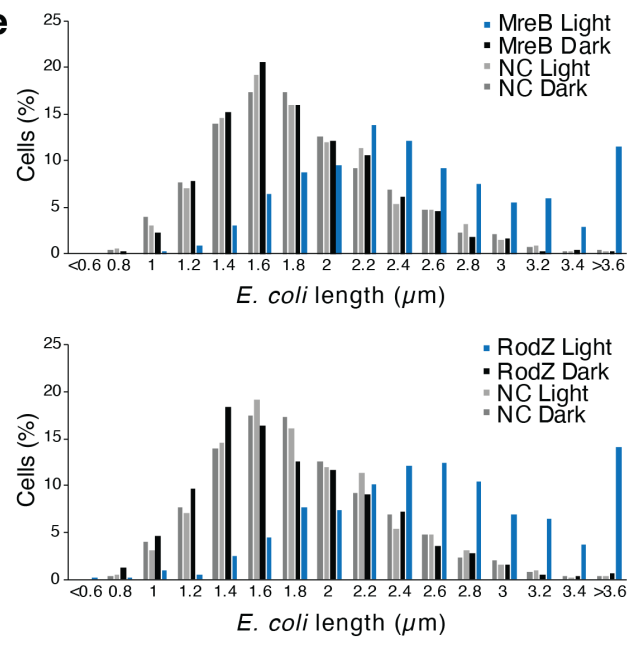

g

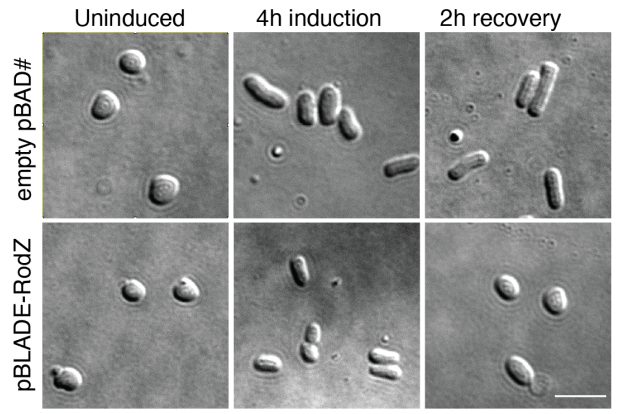

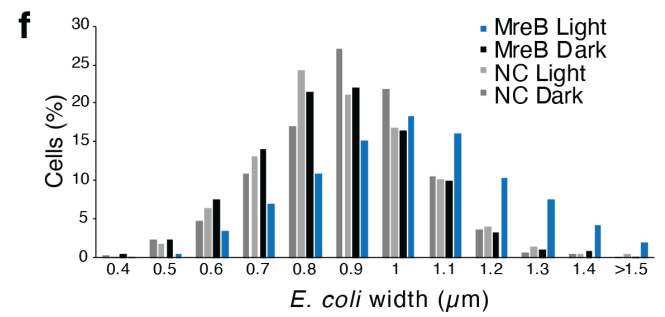

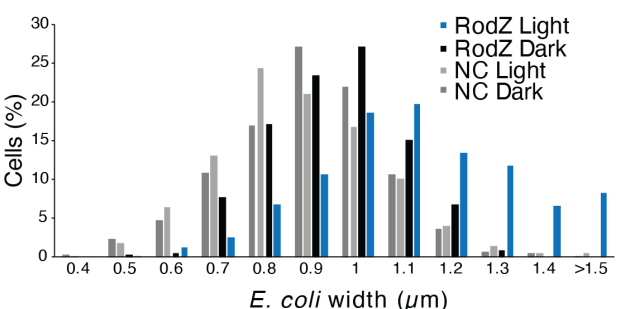

h

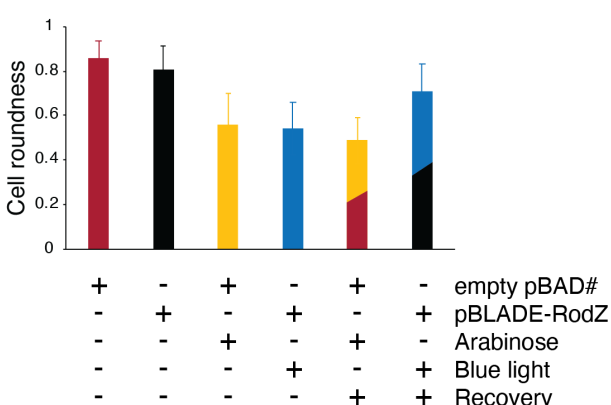

Fig. 3| BLADE enables reversible control of cell morphology. a, Phenotypes expected when overexpressing the indicated proteins. b,d, Representative DIC images of E. coli MG1655 cells transformed with the indicated constructs grown for $4 \mathrm{~h}$ either in the dark or under $460 \mathrm{~nm}$ light $\left(5 \mathrm{~W} / \mathrm{m}^{2}\right)$ illumination. c,e,f, Quantification of cell length distribution for the indicated samples and conditions. NC, pBLADE-empty. g, Representative DIC images of E. coli KC717 cells transformed with the indicated constructs at the indicated time points. Induction indicates $460 \mathrm{~nm}$ light $\left(5 \mathrm{~W} / \mathrm{m}^{2}\right)$ for the cells transformed with pBLADE-RodZ and $0.2 \%$ arabinose for the cells transformed with an empty pBAD33 from which the PBAD promoter was removed (pBAD\#). Recovery indicates darkness for the cells transformed with pBLADE-RodZ and growth in a medium without arabinose for the cells transformed with pBAD\#. h, Quantification of the cell roundness for the samples and conditions in (g). b,d,g, Scale bar, $5 \mathrm{~mm}$. c,e,f,g, Values represent mean \pm s.d. of $n=3$ independent experiments. b-h, BLADE variant: FP4 driven by the $\mathrm{J} 23101^{* *}$ promoter. 
a

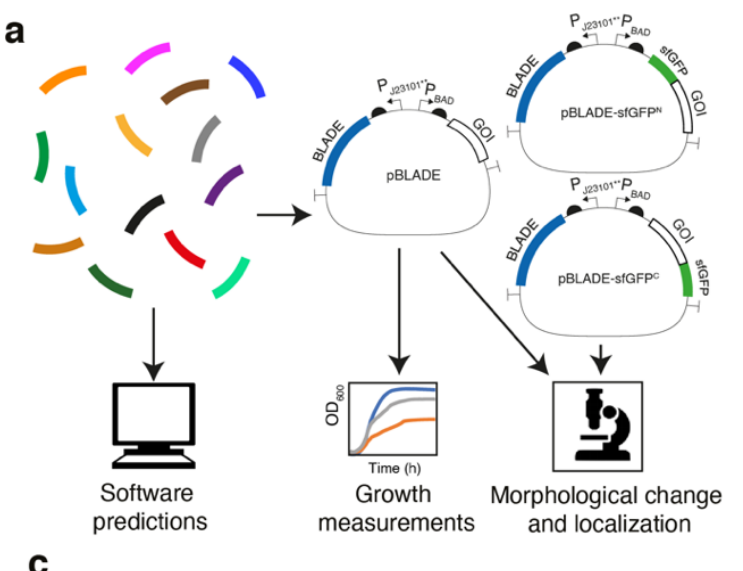

b

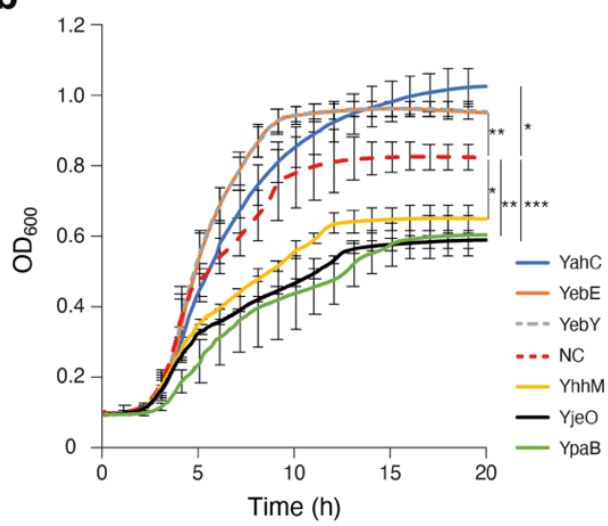

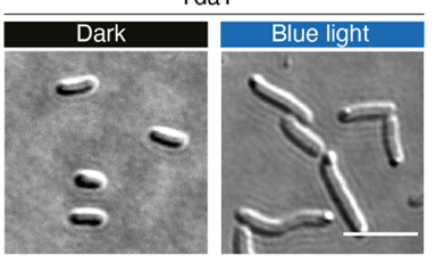

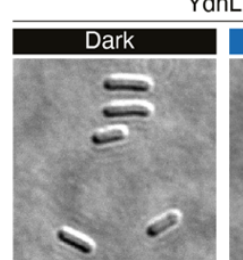

d

DIC

sfGFP

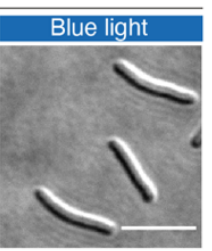

Dye

Merge

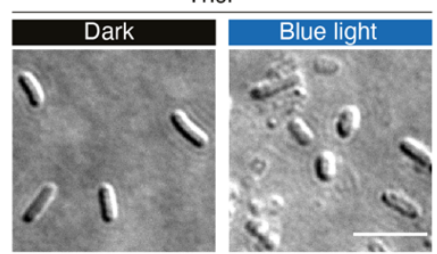

Protein with same localization
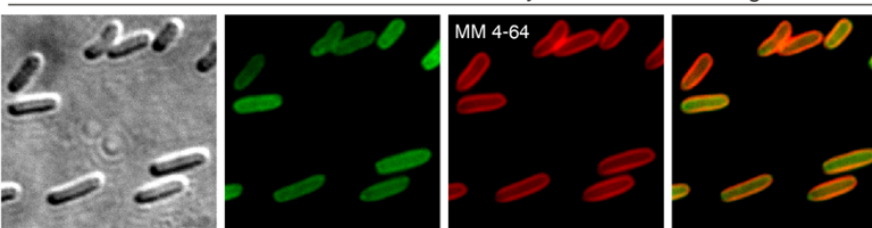

Yahc YjeO

YbaA (C) Ynau (N)

YdiY (C) YbaP

YeaO (C) YeeP

YebY (C) YehS (N)

YhcF (C) YzgL (N)

YhcF (C)
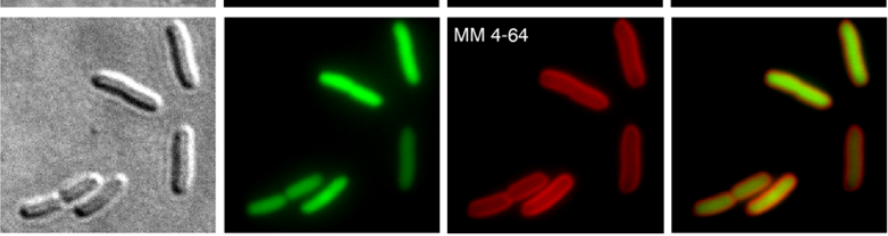

$\begin{array}{llll}\text { YggL } & \text { YdiY (N) } & \text { YggU } & \text { Yehs } \\ \text { YaiY } & \text { YdiZ } & \text { YhcF (N) } & \text { YfbM }\end{array}$

YbaA YeaO YjeO (C) YfdL

YbcV YebE YfnD YraN

YcbK YebY(N) YnaJ YzgL

YdaT(N) YegP $\quad$ YbaP(C) YibG

YdhL YfjD YnaA YpaB
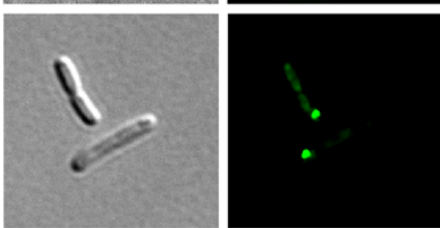

MM 4-64

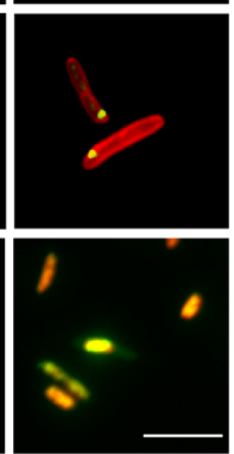

YdiL YahE YeeP

YdfR YdiL (N) YhhL YzgL (C)

YbaA (C) YdiY (C) YhhM YibG

$\mathrm{YbcV}(\mathrm{N}) \quad \mathrm{YeaO}(\mathrm{C}) \quad \mathrm{YjeO}(\mathrm{N})$

YcbK (C) YebE YnaJ (C)

$\mathrm{YceH}$ (C) YebY (C) YbaP

YceQ YegP YnaA

YahE (N) YfjD $\quad$ YfdL (N)
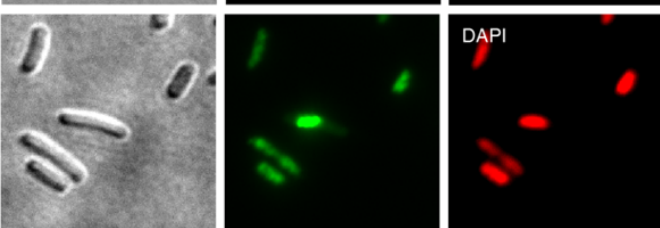

YdhL YhcF

Fig. 4| BLADE facilitates the characterization of $E$. coli genes with unknown or poorly defined

function. a, Overview of the workflow. pBLADE-sfGFP ${ }^{\mathrm{N}}$, plasmid for sfGFP N-terminal fusion; pBLADE-sfGFPC ${ }^{\mathrm{C}}$, plasmid for sfGFP C-terminal fusion. b, Growth curves of E. coli MG1655 cells transformed with pBLADE carrying the indicated proteins. NC, cells transformed with the empty plasmid. Values represent mean \pm s.d. of $n=3$ independent experiments. Single asterisk $(*)$, p-value $<0.5$ (two-tailed, homoscedastic Student's $t$ test); double asterisk $(* *)$, p-value $<0.01$ (two-tailed, homoscedastic Student's $t$ test); triple asterisk $(* * *)$, p-value $<0.001$ (two-tailed, homoscedastic Student's $t$ test). c, Representative DIC images of $E$. coli MG1655 cells transformed with pBLADE carrying the indicated proteins grown for $4 \mathrm{~h}$ either in the dark or under $460 \mathrm{~nm}$ light $\left(5 \mathrm{~W} / \mathrm{m}^{2}\right)$ illumination. d, Representative images of E. coli MG1655 cells transformed with pBLADE-sfGFP ${ }^{\mathrm{N}}$ carrying the proteins indicated in bold in the right-most column grown for $4 \mathrm{~h}$ under $460 \mathrm{~nm}$ light $(5$ $\mathrm{W} / \mathrm{m}^{2}$ ) illumination. (N), localization obtained only with $\mathrm{N}$-terminal fusion; (C), localization obtained 
a

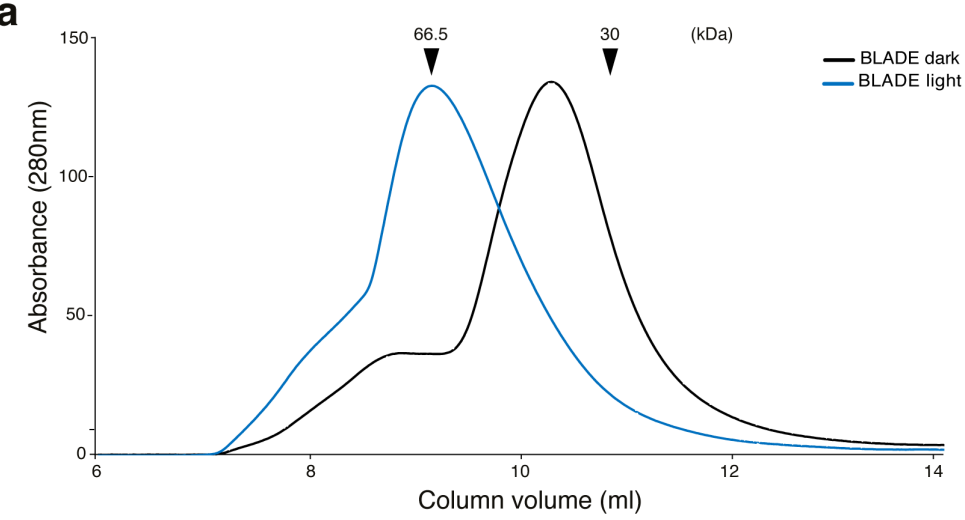

b

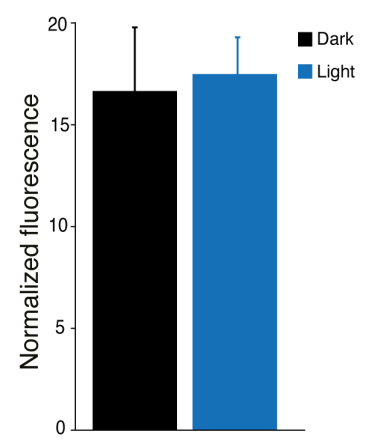

C
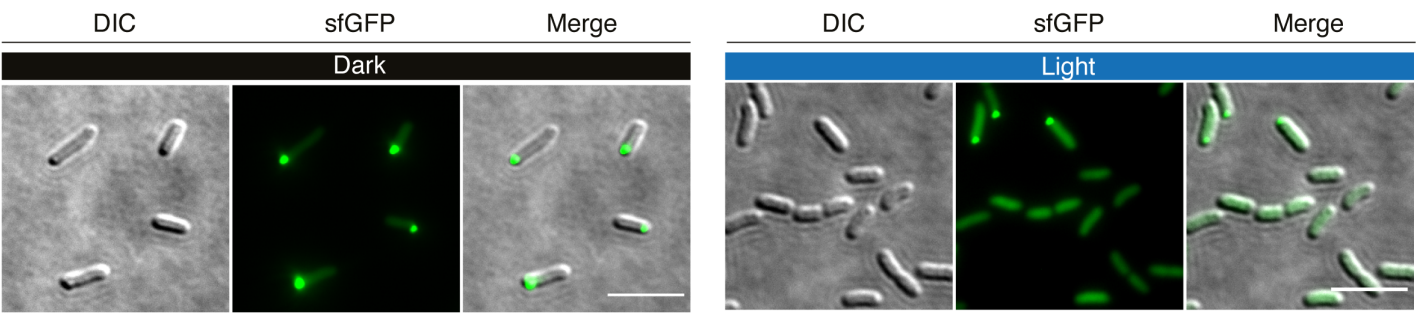

d BLADE-sfGFPC108A
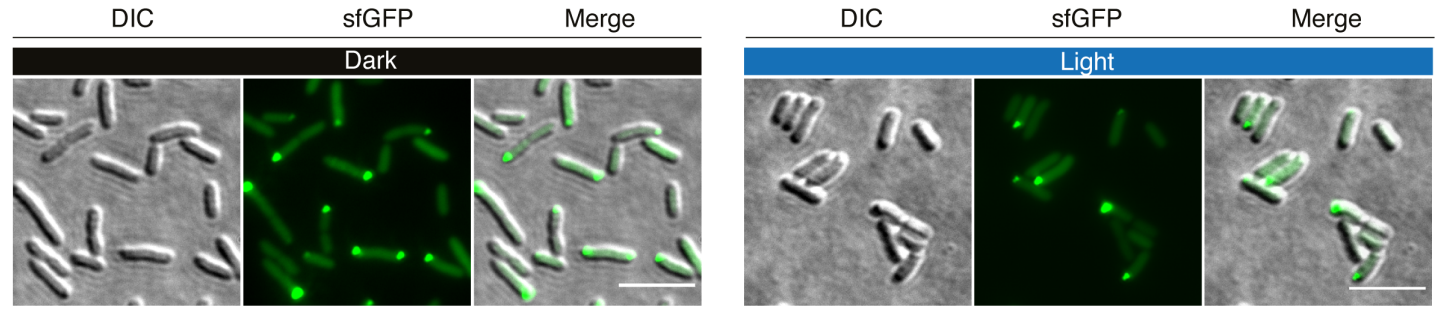

Fig. 5| BLADE-mediated light-induced gene expression involves the formation of aggregates in the dark and of dimers in the light. a, SEC performed with purified BLADE in the dark or illuminated with $460 \mathrm{~nm}$ light $\left(5 \mathrm{~W} / \mathrm{m}^{2}\right)$ for 30 minutes at $4^{\circ} \mathrm{C}$. b. GFP fluorescence intensity measured in $E$. coli MG1655 cells transformed with a modified pBLADE in which BLADE was C-terminally fused with sfGFP grown for $4 \mathrm{~h}$ in the dark or under $460 \mathrm{~nm}$ light $\left(5 \mathrm{~W} / \mathrm{m}^{2}\right)$ light. $\mathbf{c}, \mathbf{d}$, Representative microscopy images of E. coli MG1655 cells expressing the indicated BLADE variant C-terminally fused to sfGFP grown for $4 \mathrm{~h}$ in the dark or under $460 \mathrm{~nm}$ light $\left(5 \mathrm{~W} / \mathrm{m}^{2}\right)$ light. Scale bar, $5 \mu \mathrm{m}$. 
a Step 1: Find optimal BLADE expression
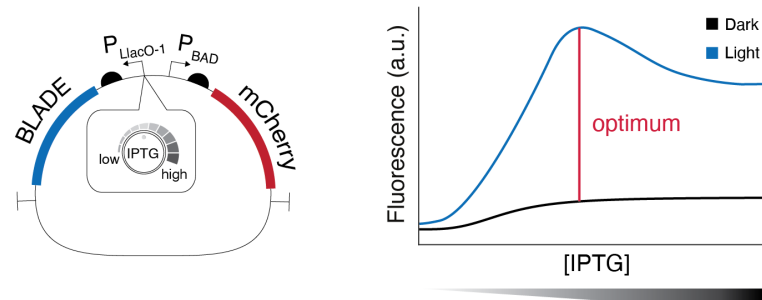

Step 2: Fix optimal BLADE expression
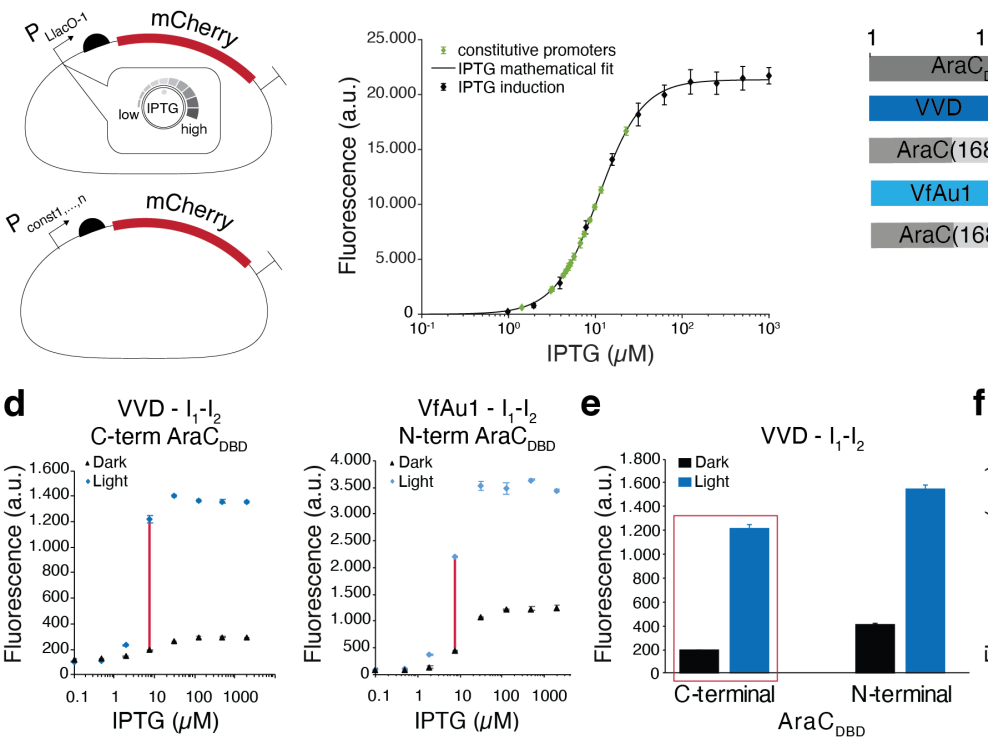

f

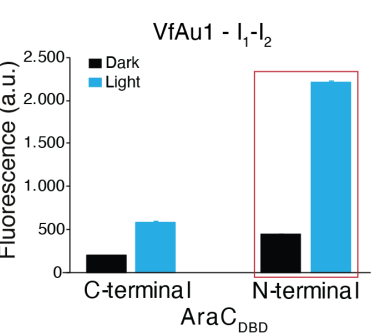

g

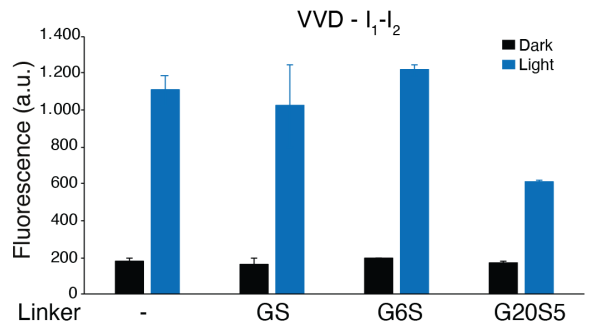

h

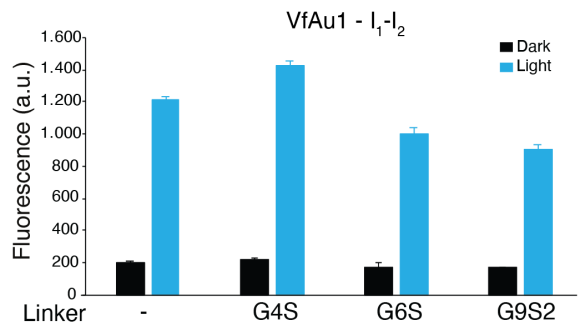

i
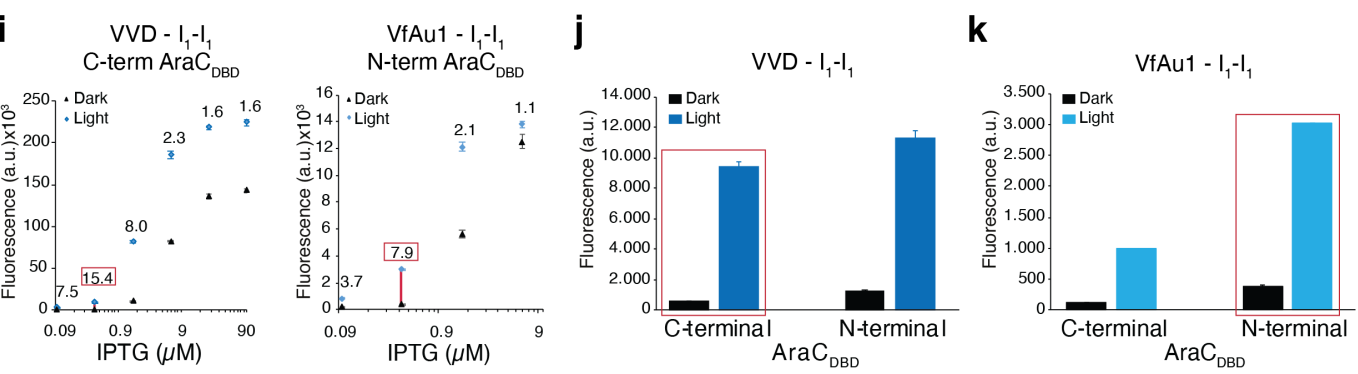

Fig. 6 Engineering an optimized and expanded family of BLADE TFs. a, A two-step protocol was used to optimize the expression levels of the TFs. Upper panel: left, the plasmid used in step 1 contains IPTG-inducible chimeric BLADE transcription factors (cTFs) and an mCherry reporter under the PBAD promoter. Upstream regulatory sequences $\left(\mathrm{O}_{1}\right.$ and $\mathrm{O}_{2}$ half-sites $)$ have been deleted. Each IPTG concentration induces a different BLADE cTF level with a corresponding light/dark mCherry expression profile. Measuring these profiles at varying IPTG concentrations allows for the identification of a profile that is optimal with respect to a desired property. Right, example of a scenario for which the highest mCherry levels are obtained at intermediate cTF levels (red line). Bottom panel: In step 2, the range of transcriptional rates induced by different IPTG concentrations is mapped to the rates of constitutive promoters in a library (right), allowing for the identification of a constitutive promoter that matches the desired optimal BLADE expression level. b, Light induction setup for 96-well microtiter plates 
containing a panel of 96 light emitting diodes (LEDs), 3D-printed holders and a metal plate for heat dissipation. $\mathbf{c}$, Domain composition of the engineered light-inducible dimerization domain (VVD and VfAu1)-AraC $C_{D B D}$ fusion constructs. d, Examples of IPTG dose-response curves obtained with BW25113 AaraC +lacYA177C cells transformed with the indicated constructs (VVD::G6S::AraCDBD and $\left.\operatorname{AraC}_{\mathrm{DBD}}::\left(\mathrm{G}_{4} \mathrm{~S}\right)_{5}:: \mathrm{VfAu} 1\right)$. The highest light/dark fold change is indicated with a red line between the corresponding data points. e, mCherry fluorescence intensity in BW25113 araC +lacYA177C cells transformed with the VVD:: $\mathrm{G}_{6} \mathrm{~S}::$ AraC $\mathrm{C}_{\text {DBD }}$ fusion (N-terminal) and the $\mathrm{AraC}_{\mathrm{DBD}}:: \mathrm{G}_{4} \mathrm{~S}:: \mathrm{VVD}$ fusion $(\mathrm{C}-$ terminal) in the presence of $7.81 \mu \mathrm{M}$ IPTG. The samples in the red box are taken from the dose response curve shown in (d). The IPTG dose-response curve of $\mathrm{AraC}_{\mathrm{DBD}}:: \mathrm{G}_{4} \mathrm{~S}:: \mathrm{VVD}$ is shown in Supplementary Fig. 14. f, mCherry fluorescence intensity in BW25113 araC +lacYA177C cells transformed with the

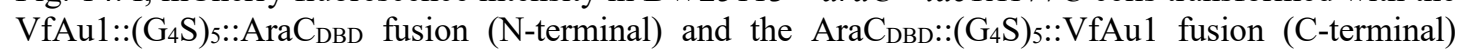
grown in the presence of $7.81 \mu \mathrm{M}$ IPTG. The samples in the red box are taken from the dose response curve shown in (d). The IPTG dose-response curve of VfAu1::(G4S) $)_{:: A r a C D B D}$ is shown in Supplementary Fig. 15. g, mCherry fluorescence intensity in BW25113 araC +lacYA177C cells transformed with a small library of C-terminal AraCDBD fusions with VVD with the indicated linkers between the two domains. The corresponding IPTG dose-response curves are shown in Supplementary Fig. 14. h, mCherry fluorescence intensity in BW25113 $\triangle$ araC + lacYA177C cells transformed with a small library of C-terminal $\mathrm{AraC}_{\mathrm{DBD}}$ fusions with VfAul with the indicated linkers between the two domains. The corresponding IPTG dose-response curves are shown in Supplementary Fig. 15. i, Same as in (d) but with a synthetic $\mathrm{P}_{\mathrm{BAD}}$ promoter containing two copies of the $\mathrm{I}_{1}$ half-site. The highest light/dark fold change is indicated with a red line between the corresponding data points. $\mathbf{j}$, Same as in (e) but with a synthetic $P_{B A D}$ promoter containing two copies of the $I_{1}$ half-site and $0.391 \mu \mathrm{M}$ IPTG. The IPTG dose-response curve of $\mathrm{AraC}_{\mathrm{DBD}}:: \mathrm{G}_{4} \mathrm{~S}:: \mathrm{VVD}$ is shown in Supplementary Fig. 16. k, Same as in (f) but with a synthetic $\mathrm{P}_{\mathrm{BAD}}$ promoter containing two copies of the $\mathrm{I}_{1}$ half-site and $0.391 \mu \mathrm{M}$ IPTG. The samples in the red box are taken from the dose response curve shown in (i). The IPTG dose-response curve of VfAu1::($\left(\mathrm{G}_{4} \mathrm{~S}\right)_{5}:: \mathrm{AraC}_{\mathrm{DBD}}$ is shown in Supplementary Fig. 17. All values represent mean \pm s.d. of $n=2$ or 3 independent experiments. In all experiments, cells were grown for $5 \mathrm{~h}$ either in the dark or under $460 \mathrm{~nm}$ light illumination. 


\section{References}

1241 1. Bren, A. et al. Glucose becomes one of the worst carbon sources for E.coli on poor nitrogen sources due to suboptimal levels of cAMP. Sci Rep 6, 24834 (2016).

2. Monod, J. Recherches sur la croissance des cultures bacttriennes. Band 911 von Actualités scientifiques et industrielles, (1942).

3. Schleif, R. Regulation of the L-arabinose operon of Escherichia coli. Trends Genet 16, 559-565 (2000).

4. Schleif, R. AraC protein, regulation of the 1-arabinose operon in Escherichia coli, and the light switch mechanism of AraC action. FEMS Microbiol Rev 34, 779-796 (2010).

5. Wang, X., Chen, X. \& Yang, Y. Spatiotemporal control of gene expression by a light-switchable transgene system. Nat Methods 9, 266-269 (2012).

6. Chen, X. et al. An extraordinary stringent and sensitive light-switchable gene expression system for bacterial cells. Cell Res 26, 854-857 (2016).

7. Colavin, A., Shi, H. \& Huang, K.C. RodZ modulates geometric localization of the bacterial actin MreB to regulate cell shape. Nat Commun 9, 1280 (2018).

8. Rengby, O. \& Arner, E.S. Titration and conditional knockdown of the prfB gene in Escherichia coli: effects on growth and overproduction of the recombinant mammalian selenoprotein thioredoxin reductase. Appl Environ Microbiol 73, 432-441 (2007).

9. Roux, A., Beloin, C. \& Ghigo, J.M. Combined inactivation and expression strategy to study gene function under physiological conditions: application to identification of new Escherichia coli adhesins. J Bacteriol 187, 1001-1013 (2005).

10. Schwerdtfeger, C. \& Linden, H. VIVID is a flavoprotein and serves as a fungal blue light photoreceptor for photoadaptation. EMBO J 22, 4846-4855 (2003).

11. Bustos, S.A. \& Schleif, R.F. Functional domains of the AraC protein. Proc Natl Acad Sci U S A 90, 5638-5642 (1993).

12. Timmes, A., Rodgers, M. \& Schleif, R. Biochemical and physiological properties of the DNA binding domain of AraC protein. J Mol Biol 340, 731738 (2004).

13. Sheets, M.B., Wong, W.W. \& Dunlop, M.J. Light-Inducible Recombinases for Bacterial Optogenetics. ACS Synth Biol 9, 227-235 (2020).

14. $\mathrm{Xu}, \mathrm{X}$. et al. A Single-Component Optogenetic System Allows Stringent Switch of Gene Expression in Yeast Cells. ACS Synth Biol 7, 2045-2053 (2018).

15. Vaidya, A.T., Chen, C.H., Dunlap, J.C., Loros, J.J. \& Crane, B.R. Structure of a light-activated LOV protein dimer that regulates transcription. Sci Signal 4, ra50 (2011).

16. Zoltowski, B.D. \& Crane, B.R. Light activation of the LOV protein vivid generates a rapidly exchanging dimer. Biochemistry 47, 7012-7019 (2008).

17. de Boer, P.A., Crossley, R.E. \& Rothfield, L.I. A division inhibitor and a topological specificity factor coded for by the minicell locus determine proper placement of the division septum in E. coli. Cell 56, 641-649 (1989).

18. Di Ventura, B. et al. Chromosome segregation by the Escherichia coli Min system. Mol Syst Biol 9, 686 (2013). 
19. de Boer, P.A., Crossley, R.E. \& Rothfield, L.I. Roles of MinC and MinD in the site-specific septation block mediated by the MinCDE system of Escherichia coli. J Bacteriol 174, 63-70 (1992).

20. Hu, Z., Mukherjee, A., Pichoff, S. \& Lutkenhaus, J. The MinC component of the division site selection system in Escherichia coli interacts with FtsZ to prevent polymerization. Proc Natl Acad Sci U S A 96, 14819-14824 (1999).

21. Raskin, D.M. \& de Boer, P.A. Rapid pole-to-pole oscillation of a protein required for directing division to the middle of Escherichia coli. Proc Natl Acad Sci U S A 96, 4971-4976 (1999).

22. Pedelacq, J.D., Cabantous, S., Tran, T., Terwilliger, T.C. \& Waldo, G.S. Engineering and characterization of a superfolder green fluorescent protein. Nat Biotechnol 24, 79-88 (2006).

23. Levskaya, A. et al. Synthetic biology: engineering Escherichia coli to see light. Nature 438, 441-442 (2005).

24. Yang, D.C., Blair, K.M. \& Salama, N.R. Staying in Shape: the Impact of Cell Shape on Bacterial Survival in Diverse Environments. Microbiol Mol Biol Rev 80, 187-203 (2016).

25. Smith, W.P. et al. Cell morphology drives spatial patterning in microbial communities. Proc Natl Acad Sci U S A 114, E280-E286 (2017).

26. $\mathrm{Hu}, \mathrm{Z} . \&$ Lutkenhaus, J. A conserved sequence at the C-terminus of MinD is required for binding to the membrane and targeting MinC to the septum. Mol Microbiol 47, 345-355 (2003).

27. Szeto, T.H., Rowland, S.L., Habrukowich, C.L. \& King, G.F. The MinD membrane targeting sequence is a transplantable lipid-binding helix. $J$ Biol Chem 278, 40050-40056 (2003).

28. Wachi, M. et al. Mutant isolation and molecular cloning of mre genes, which determine cell shape, sensitivity to mecillinam, and amount of penicillinbinding proteins in Escherichia coli. J Bacteriol 169, 4935-4940 (1987).

29. Doi, M. et al. Determinations of the DNA sequence of the mreB gene and of the gene products of the mre region that function in formation of the rod shape of Escherichia coli cells. J Bacteriol 170, 4619-4624 (1988).

30. Wachi, M. \& Matsuhashi, M. Negative control of cell division by mreB, a gene that functions in determining the rod shape of Escherichia coli cells. $J$ Bacteriol 171, 3123-3127 (1989).

31. Bendezu, F.O., Hale, C.A., Bernhardt, T.G. \& de Boer, P.A. RodZ (YfgA) is required for proper assembly of the MreB actin cytoskeleton and cell shape in E. coli. EMBO J 28, 193-204 (2009).

32. Alyahya, S.A. et al. RodZ, a component of the bacterial core morphogenic apparatus. Proc Natl Acad Sci U S A 106, 1239-1244 (2009).

33. van den Ent, F., Johnson, C.M., Persons, L., de Boer, P. \& Lowe, J. Bacterial actin MreB assembles in complex with cell shape protein RodZ. EMBO J 29, 1081-1090 (2010).

34. Kruse, T., Moller-Jensen, J., Lobner-Olesen, A. \& Gerdes, K. Dysfunctional MreB inhibits chromosome segregation in Escherichia coli. EMBO J 22, 5283-5292 (2003).

35. Shiomi, D., Sakai, M. \& Niki, H. Determination of bacterial rod shape by a novel cytoskeletal membrane protein. EMBO J 27, 3081-3091 (2008).

36. Ghatak, S., King, Z.A., Sastry, A. \& Palsson, B.O. The y-ome defines the $35 \%$ of Escherichia coli genes that lack experimental evidence of function. Nucleic Acids Res 47, 2446-2454 (2019). 
37. Bindal, G., Krishnamurthi, R., Seshasayee, A.S.N. \& Rath, D. CRISPR-CasMediated Gene Silencing Reveals RacR To Be a Negative Regulator of YdaS and YdaT Toxins in Escherichia coli K-12. mSphere 2 (2017).

38. Stancik, L.M. et al. pH-dependent expression of periplasmic proteins and amino acid catabolism in Escherichia coli. J Bacteriol 184, 4246-4258 (2002).

39. Chodisetti, P.K. \& Reddy, M. Peptidoglycan hydrolase of an unusual crosslink cleavage specificity contributes to bacterial cell wall synthesis. Proc Natl Acad Sci U S A 116, 7825-7830 (2019).

40. Otoupal, P.B. \& Chatterjee, A. CRISPR Gene Perturbations Provide Insights for Improving Bacterial Biofuel Tolerance. Front Bioeng Biotechnol 6, 122 (2018).

41. Yamamoto, K. \& Ishihama, A. Characterization of copper-inducible promoters regulated by $\mathrm{CpxA} / \mathrm{CpxR}$ in Escherichia coli. Biosci Biotechnol Biochem 70, 1688-1695 (2006).

42. Raivio, T.L., Leblanc, S.K. \& Price, N.L. The Escherichia coli Cpx envelope stress response regulates genes of diverse function that impact antibiotic resistance and membrane integrity. J Bacteriol 195, 2755-2767 (2013).

43. Brieger, A. et al. C-terminal fluorescent labeling impairs functionality of DNA mismatch repair proteins. PLoS One 7, e31863 (2012).

44. Shiomi, D. \& Margolin, W. The C-terminal domain of MinC inhibits assembly of the $\mathrm{Z}$ ring in Escherichia coli. J Bacteriol 189, 236-243 (2007).

45. Weill, U. et al. Assessment of GFP Tag Position on Protein Localization and Growth Fitness in Yeast. J Mol Biol 431, 636-641 (2019).

46. Falda, M. et al. Argot2: a large scale function prediction tool relying on semantic similarity of weighted Gene Ontology terms. BMC Bioinformatics 13 Suppl 4, S14 (2012).

47. Toronen, P., Medlar, A. \& Holm, L. PANNZER2: a rapid functional annotation web server. Nucleic Acids Res 46, W84-W88 (2018).

48. Kulmanov, M. \& Hoehndorf, R. DeepGOPlus: improved protein function prediction from sequence. Bioinformatics 36, $422-429$ (2020).

49. Kelley, L.A., Mezulis, S., Yates, C.M., Wass, M.N. \& Sternberg, M.J. The Phyre2 web portal for protein modeling, prediction and analysis. Nat Protoc 10, 845-858 (2015).

50. Yuan, J. et al. Vibrio cholerae ParE2 poisons DNA gyrase via a mechanism distinct from other gyrase inhibitors. J Biol Chem 285, 40397-40408 (2010).

51. Hallez, R. et al. New toxins homologous to ParE belonging to threecomponent toxin-antitoxin systems in Escherichia coli O157:H7. Mol Microbiol 76, 719-732 (2010).

52. Lee, N., Francklyn, C. \& Hamilton, E.P. Arabinose-induced binding of AraC protein to araI2 activates the araBAD operon promoter. Proc Natl Acad Sci U $S$ A 84, 8814-8818 (1987).

53. Malzahn, E., Ciprianidis, S., Kaldi, K., Schafmeier, T. \& Brunner, M. Photoadaptation in Neurospora by competitive interaction of activating and inhibitory LOV domains. Cell 142, 762-772 (2010).

54. Heintzen, C., Loros, J.J. \& Dunlap, J.C. The PAS protein VIVID defines a clock-associated feedback loop that represses light input, modulates gating, and regulates clock resetting. Cell 104, 453-464 (2001).

55. Hunt, S.M., Elvin, M., Crosthwaite, S.K. \& Heintzen, C. The PAS/LOV protein VIVID controls temperature compensation of circadian clock phase and development in Neurospora crassa. Genes Dev 21, 1964-1974 (2007). 
1388

1389

1390

1391

1392

1393

1394

1395

1396

1397

1398

1399

1400

1401

1402

1403

1404

1405

1406

1407

1408

1409

1410

1411

1412

1413

1414

1415

1416

1417

1418

1419

1420

1421

1422

1423

1424

1425

1426

1427

1428

1429

1430

1431

1432

1433

1434

1435

1436

56. Lee, C.T., Malzahn, E., Brunner, M. \& Mayer, M.P. Light-induced differences in conformational dynamics of the circadian clock regulator VIVID. $J \mathrm{Mol}$ Biol 426, 601-610 (2014).

57. Mitra, D., Yang, X. \& Moffat, K. Crystal structures of Aureochrome1 LOV suggest new design strategies for optogenetics. Structure 20, 698-706 (2012).

58. Takahashi, F. et al. AUREOCHROME, a photoreceptor required for photomorphogenesis in stramenopiles. Proc Natl Acad Sci U S A 104, 1962519630 (2007).

59. Toyooka, T., Hisatomi, O., Takahashi, F., Kataoka, H. \& Terazima, M. Photoreactions of aureochrome-1. Biophys J 100, 2801-2809 (2011).

60. Grusch, M. et al. Spatio-temporally precise activation of engineered receptor tyrosine kinases by light. EMBO J 33, 1713-1726 (2014).

61. Baumschlager, A., Aoki, S.K. \& Khammash, M. Dynamic Blue LightInducible T7 RNA Polymerases (Opto-T7RNAPs) for Precise Spatiotemporal Gene Expression Control. ACS Synth Biol 6, 2157-2167 (2017).

62. Lutz, R. \& Bujard, H. Independent and tight regulation of transcriptional units in Escherichia coli via the $\mathrm{LacR} / \mathrm{O}$, the TetR/O and AraC/I1-I2 regulatory elements. Nucleic Acids Res 25, 1203-1210 (1997).

63. Baba, T. et al. Construction of Escherichia coli K-12 in-frame, single-gene knockout mutants: the Keio collection. Mol Syst Biol 2, 20060008 (2006).

64. Chen, X., Zaro, J.L. \& Shen, W.C. Fusion protein linkers: property, design and functionality. Adv Drug Deliv Rev 65, 1357-1369 (2013).

65. http://parts.igem.org/Promoters/Catalog/Anderson.

66. Lou, C., Stanton, B., Chen, Y.J., Munsky, B. \& Voigt, C.A. Ribozyme-based insulator parts buffer synthetic circuits from genetic context. Nat Biotechnol 30, 1137-1142 (2012).

67. Reeder, T. \& Schleif, R. AraC protein can activate transcription from only one position and when pointed in only one direction. J Mol Biol 231, 205-218 (1993).

68. Ohlendorf, R., Vidavski, R.R., Eldar, A., Moffat, K. \& Moglich, A. From dusk till dawn: one-plasmid systems for light-regulated gene expression. $J \mathrm{Mol}$ Biol 416, 534-542 (2012).

69. Ramakrishnan, P. \& Tabor, J.J. Repurposing Synechocystis PCC6803 UirSUirR as a UV-Violet/Green Photoreversible Transcriptional Regulatory Tool in E. coli. ACS Synth Biol 5, 733-740 (2016).

70. Ong, N.T. \& Tabor, J.J. A Miniaturized Escherichia coli Green Light Sensor with High Dynamic Range. Chembiochem 19, 1255-1258 (2018).

71. Jayaraman, P. et al. Blue light-mediated transcriptional activation and repression of gene expression in bacteria. Nucleic Acids Res 44, 6994-7005 (2016).

72. Li, X. et al. A single-component light sensor system allows highly tunable and direct activation of gene expression in bacterial cells. Nucleic Acids Res $\mathbf{4 8}$, e33 (2020).

73. Siegele, D.A. \& Hu, J.C. Gene expression from plasmids containing the araBAD promoter at subsaturating inducer concentrations represents mixed populations. Proc Natl Acad Sci U S A 94, 8168-8172 (1997).

74. Khlebnikov, A., Risa, O., Skaug, T., Carrier, T.A. \& Keasling, J.D. Regulatable arabinose-inducible gene expression system with consistent control in all cells of a culture. J Bacteriol 182, 7029-7034 (2000). 
1437

1438

1439

1440

1441

1442

1443

1444

1445

1446

1447

1448

1449

1450

1451

1452

1453

1454

1455

1456

1457

1458

1459

1460

1461

1462

1463

1464

1465

1466

1467

1468

1469

1470

1471

1472

75. Morgan-Kiss, R.M., Wadler, C. \& Cronan, J.E., Jr. Long-term and homogeneous regulation of the Escherichia coli araBAD promoter by use of a lactose transporter of relaxed specificity. Proc Natl Acad Sci U S A 99, 73737377 (2002).

76. Hernandez-Candia, C.N., Casas-Flores, S. \& Gutierrez-Medina, B. Light induces oxidative damage and protein stability in the fungal photoreceptor Vivid. PLoS One 13, e0201028 (2018).

77. O'Connell, J.D., Zhao, A., Ellington, A.D. \& Marcotte, E.M. Dynamic reorganization of metabolic enzymes into intracellular bodies. Annu Rev Cell Dev Biol 28, 89-111 (2012).

78. Lindner, A.B., Madden, R., Demarez, A., Stewart, E.J. \& Taddei, F. Asymmetric segregation of protein aggregates is associated with cellular aging and rejuvenation. Proc Natl Acad Sci U S A 105, 3076-3081 (2008).

79. Benzinger, D. \& Khammash, M. Pulsatile inputs achieve tunable attenuation of gene expression variability and graded multi-gene regulation. Nat Commun 9, 3521 (2018).

80. Zhao, E.M. et al. Optogenetic regulation of engineered cellular metabolism for microbial chemical production. Nature 555, 683-687 (2018).

81. Datsenko, K.A. \& Wanner, B.L. One-step inactivation of chromosomal genes in Escherichia coli K-12 using PCR products. Proc Natl Acad Sci U S A 97, 6640-6645 (2000).

82. Bowers, L.M., Lapoint, K., Anthony, L., Pluciennik, A. \& Filutowicz, M. Bacterial expression system with tightly regulated gene expression and plasmid copy number. Gene 340, 11-18 (2004).

83. Kentner, D., Thiem, S., Hildenbeutel, M. \& Sourjik, V. Determinants of chemoreceptor cluster formation in Escherichia coli. Mol Microbiol 61, 407417 (2006).

84. Thiem, S., Kentner, D. \& Sourjik, V. Positioning of chemosensory clusters in E. coli and its relation to cell division. EMBO J 26, 1615-1623 (2007).

85. Potapov, V. et al. Comprehensive Profiling of Four Base Overhang Ligation Fidelity by T4 DNA Ligase and Application to DNA Assembly. ACS Synth Biol 7, 2665-2674 (2018).

86. Chung, C.T., Niemela, S.L. \& Miller, R.H. One-step preparation of competent Escherichia coli: transformation and storage of bacterial cells in the same solution. Proc Natl Acad Sci U S A 86, 2172-2175 (1989). 